\title{
Discrete growth-decay-fragmentation equation: well-posedness and long-term dynamics
}

\author{
J. BANASIAK, L.O. JOEL AND S. SHINDIN
}

Abstract. In this paper, we consider discrete growth-decay-fragmentation equations that describe the size distribution of clusters that can undergo splitting, growth and decay. The clusters can be for instance animal groups that can split but can also grow, or decrease in size due to birth or death of individuals in the group, or chemical particles where the growth and decay can be due to surface deposition or erosion. We prove that for a large class of such problems, the solution semigroup is analytic and compact and thus has the asynchronous exponential growth property; that is, the long-term behaviour of any solution is given by a scalar exponential function multiplied by a vector, called the stable population distribution, that is independent on the initial conditions.

\section{Introduction}

Coagulation and fragmentation models that describe the processes of objects forming larger clusters or, conversely, splitting into smaller fragments have received a lot of attention over several decades due to their importance in chemical engineering and other fields of science and technology, see, e.g., [21,42]. One of the most efficient approaches to modelling dynamics of such processes is through the kinetic (rate) equation which describes the evolution of the distribution of interacting clusters with respect to their size/mass. The first model of this kind, consisting of an infinite system of ordinary differential equations, was derived by Smoluchowski [40,41], to describe pure coagulation in the discrete case, that is, if the ratio of the mass of the basic building block (monomer) to the mass of a typical cluster is positive, and thus the size of a cluster is a finite multiple of the masses of the monomers. In many applications, however, it turned out to be advantageous to allow clusters to be composed of particles of any size $x>0$. This leads to the continuous integro-differential equation that was derived by Müller in the pure coagulation case [32] and extended to a coagulation-fragmentation version in [29].

Mathematics Subject Classification: 34G10, 35B40, 35P05, 47D06, 45K05, 80A30

Keywords: Discrete fragmentation, Birth-and-death process, $C_{0}$-Semigroups, Long-term behaviour, Asynchronous exponential growth, Spectral gap, Numerical simulations.

The research was partially funded by DST/NRF SARChI Chair in Mathematical Models and Methods in Biosciences and Bioengineering, Grant No. 8277, and NRF Ph.D. Grantholder Bursary, Grant No. 102275 (LOJ). 
In the last few decades, it has been observed that also living organisms form clusters or split into subgroups depending on circumstances, see, e.g., [17,26,33,34] for modelling concerning larger animals or [1,27] for phytoplankton models. It turns out that also the process of cell division may be modelled within the same framework, see, e.g., $[12,13,36,38]$. What was not always fully recognized in some papers mentioned above was that the living matter has its own vital dynamics; that is, in addition to forming or breaking clusters, individuals within them are born or die, leading to the growth or decay of the clusters, and so the latter processes must be adequately represented in the models. In the continuous case, the birth-and-death processes are incorporated into the model by adding an appropriate first-order transport term, analogously to the ageor size-structured McKendrick model, see $[1,7,9,12,36]$. On the other hand, in the discrete case, the vital processes are modelled by adding the classical birth-and-death terms to the Smoluchowski equation. Note that, e.g., the pure birth terms (or pure death terms) can be obtained by the Euler discretization of the first-order differential operator of the continuous case, while the full birth-and-death problem can be thought of as the discretization of the diffusion operator.

One of the most important problems in the analysis of dynamical systems is to determine their long-term behaviour, and hence, this aspect of the theory of growthfragmentation equations has received much attention. The first systematic mathematical study of the binary cell division model was carried out using semigroup theory in [18]; the semigroup approach was significantly extended to more general models in [31]. Recently, a number of results have been obtained by the General Relative Entropy (or related) methods that lead to convergence of solutions in spaces weighted by the eigenvector of the adjoint problem, see, e.g., [5, 19,20,28,30,36,37].

All the above results concern growth-fragmentation models with continuous size distribution. Recently, it has been observed, however, that a large class of discrete fragmentation equations has much better properties than their continuous counterparts, especially when considered in spaces where sufficiently high moments of solutions are finite. In particular, the fragmentation operator in such spaces generates a compact analytic semigroup. In this paper, we explore these ideas for the full growth-decayfragmentation equation and show, in particular, that under natural assumptions on the coefficients of the problem, the growth-decay-fragmentation semigroup is analytic, compact and irreducible and thus has the asynchronous exponential growth (AEG) property, see [3]; that is, its long-term behaviour of solutions is given by the scalar exponential function $e^{\lambda t}$, where $\lambda$ is the dominant eigenvalue (and the spectral bound) of the fragmentation operator, multiplied by the strictly positive eigenvector belonging to this eigenvalue, called the stable population distribution. The word asynchronous comes from the fact that the behaviour of solutions is 'not synchronized' with the initial conditions.

The paper is organized as follows. In Sect. 2, we introduce the model. Sections 3 and 4 contain the results on the solvability of the problem. We use the perturbation method, and thus the way in which the right-hand side of the equation is split is of 
utmost importance. We found that first considering the subdiagonal part (Sect. 3) and treating the full equation as a perturbation of the subdiagonal part by remaining part, being a positive operator, by using the Kato perturbation theorem (Sect. 4) provides the best results. In "Appendix," we provide a description of an alternative method, in which we split the equations in a seemingly more natural way, considering the processes of growth and decay separately from the independent process of fragmentation and then using the Trotter-Kato representation formula to prove the existence of the solution semigroup. We find that this approach produces weaker results due to worse properties of the growth-decay semigroup. Finally, in Sect. 5, we show that the growth-decay-fragmentation semigroup has the AEG property and in Sect. 6 we provide some examples and numerical illustrations of the theoretical results obtained in the earlier sections.

\section{The model}

We consider a collection of clusters of sizes $n \in \mathbb{N}$, that is, consisting of $n$ monomers (cells, individuals,...), described by their size-specific density $f=\left(f_{n}\right)_{n \in \mathbb{N}}$. We assume that the number of monomers in each cluster can change by, say, a cell division (with the daughter cell staying in the cluster) or its death. In an inanimate scenario, this can happen by the deposition of a particle from the solute or, conversely, by its dissolution. If we assume that the probability of a more than one birth or death event in a cluster happening simultaneously is negligible, then the process can be modelled by the classical birth-and-death system of equations, see, e.g., [27, p. 1199], though in this setting it should be remembered that the birth or death in a cluster leads to its growth or decay and the latter is the subject of the modelling process. We note that in the case of continuous size distribution, the growth process is modelled by the first-order differential operator with respect to size, $f \rightarrow-\partial_{x}(g f)$, where $g$ is the growth rate, see, e.g., [7], whose Euler discretization with step size 1 at $x=n$ is $g(n) f(n)-g(n+1) f(n+1)$. Similarly, the decay operator $f \rightarrow \partial_{x}(d f)$ can be discretized as $-d(n) f(n)+d(n+1) f(n+1)$ and, using a central difference scheme, the diffusion operator $f \rightarrow \partial_{x}\left(D \partial_{x} f\right)$ yields $D(n+1) f(n+1)-(D(n+1)+D(n)) f(n)+D(n) f(n-1)$.

We further assume that the clusters can split into several smaller clusters. Combining both processes, we arrive at the following system of equations:

$$
\begin{aligned}
\frac{\mathrm{d} f_{1}}{\mathrm{~d} t} & =-g_{1} f_{1}+d_{2} f_{2}+\sum_{i=2}^{\infty} a_{i} b_{1, i} f_{i}, \\
\frac{\mathrm{d} f_{n}}{\mathrm{~d} t} & =g_{n-1} f_{n-1}-\left(a_{n}+g_{n}+d_{n}\right) f_{n}+d_{n+1} f_{n+1}+\sum_{i=n+1}^{\infty} a_{i} b_{n, i} f_{i}, \quad n \geq 2, \\
f_{n}(0) & =f_{n}^{\text {in }}, \quad n \geq 1,
\end{aligned}
$$


or

$$
\begin{aligned}
\frac{\mathrm{d} f}{\mathrm{~d} t} & =\mathcal{G}^{-} f+\left(\mathcal{A}+\mathcal{G}^{0}+\mathcal{D}^{0}\right) f+\mathcal{D}^{+} f+\Xi \mathcal{A} f=\mathcal{G} f+\mathcal{D} f+\mathcal{A} f+\mathcal{B} f \\
& =\mathcal{G} f+\mathcal{D} f+\mathcal{F} f \\
f(0) & =f^{\text {in }}
\end{aligned}
$$

where $f=\left(f_{n}\right)_{n \in \mathbb{N}}$ is the vector whose components $f_{n}$ give the numbers of $n$-clusters; the operator

$$
\mathcal{A}=\operatorname{diag}\left(-a_{n}\right)_{n \geq 1}, \quad a_{1}=0, \quad a_{n}>0, \quad n \geq 2,
$$

gives the rates at which the clusters of mass $n$ undergo splitting;

$$
\mathcal{G}^{0}=\operatorname{diag}\left(-g_{n}\right)_{n \geq 1}, \quad g_{n} \geq 0, \quad n \geq 1,
$$

is the growth rate;

$$
\mathcal{D}^{0}=\operatorname{diag}\left(-d_{n}\right)_{n \geq 1}, \quad d_{1}=0, \quad d_{n} \geq 0, \quad n \geq 2,
$$

is the death rate; $\mathcal{G}^{-}, \mathcal{D}^{+}$are, respectively, the left and right shifts of $\mathcal{G}$ and $\mathcal{D}$; that is

$$
\mathcal{G}^{-} f=\left(0, g_{1} f_{1}, \ldots g_{n} f_{n}, \ldots\right), \quad \mathcal{D}^{+} f=\left(d_{2} f_{2}, \ldots d_{n} f_{n}, \ldots\right),
$$

$\Xi=\left(b_{n, i}\right)_{1 \leq n<i, i \geq 2}$ is the daughter distribution function, also called the fragmentation kernel, that gives the numbers of $i$-clusters resulting from splitting of a mass $n$ parent and

$$
\mathcal{G}=\mathcal{G}^{-}+\mathcal{G}^{0}, \quad \mathcal{D}=\mathcal{D}^{+}+\mathcal{D}^{0}, \quad \mathcal{B}=\Xi \mathcal{A}, \quad \mathcal{F}=\mathcal{A}+\mathcal{B}
$$

Coefficients $b_{n, i}, 1 \leq n<i, i \geq 2$, are nonnegative numbers satisfying

$$
\sum_{n=1}^{i-1} n b_{n, i}=i .
$$

The total mass of the ensemble is given by

$$
M(t)=\sum_{n=1}^{\infty} n f_{n}(t), \quad t \geq 0
$$

then it is known, see, e.g., $[6,10]$, that in the pure fragmentation case $(\mathcal{G}=\mathcal{D}=0)$ the mass is conserved, $M(t)=\sum_{i=1}^{\infty} n f_{n}(0), t \geq 0$.

Later, we shall use the fact that (1) can be written as the pure growth-fragmentation model 


$$
\begin{aligned}
\frac{\mathrm{d} f_{1}}{\mathrm{~d} t} & =-g_{1} f_{1}+\sum_{i=2}^{\infty} \mathrm{a}_{i} \mathrm{~b}_{1, i} f_{i}, \\
\frac{\mathrm{d} f_{n}}{\mathrm{~d} t} & =g_{n-1} f_{n-1}-\left(g_{n}+\mathrm{a}_{n}\right) f_{n}+\sum_{i=n+1}^{\infty} \mathrm{a}_{i} \mathrm{~b}_{n, i} f_{i}, \quad n \geq 2, \\
f_{n}(0) & =f_{n}^{\text {in }}, \quad n \geq 1,
\end{aligned}
$$

where $\mathrm{a}_{n}=a_{n}+d_{n}, n \geq 2$, (with $\left.\mathrm{a}_{1}=0\right)$ and

$$
\mathrm{b}_{n, i}= \begin{cases}\frac{a_{n+1} b_{n, n+1}+d_{n+1}}{a_{n+1}+d_{n+1}}, & i=n+1, \\ \frac{a_{i} b_{n, i}}{a_{i}+d_{i}}, & i \geq n+2 .\end{cases}
$$

We note that the fragmentation part of this model no longer is conservative as

$$
\sum_{n=1}^{i-1} n \mathrm{~b}_{n, i}=i\left(1-\frac{d_{i}}{i\left(a_{i}+d_{i}\right)}\right), \quad i \geq 2,
$$

so it corresponds to the model with the so-called discrete mass loss with mass loss fraction $\lambda_{n}=d_{n} / n\left(a_{n}+d_{n}\right)$, see [14,22], mathematically analysed in [39].

The analysis of the pure fragmentation equation most often is carried out in the space $X_{1}:=\ell_{1}^{1}$ in which the norm of a nonnegative $f$ gives the total mass of the ensemble. However, it is much better to consider (1) in the spaces with finite higher moments, $X_{m}:=\ell_{m}^{1}$, with the norm

$$
\|f\|_{[m]}=\sum_{n=1}^{\infty} n^{m}\left|f_{n}\right|, \quad m \geq 1 .
$$

In the sequel, for any infinite diagonal matrix $\mathcal{P}=\operatorname{diag}\left(p_{n}\right)_{n \geq 1}$, we define the operator $P_{m}$ in $X_{m}$ by $P_{m} f=\mathcal{P} f$ on $D\left(P_{m}\right)=\left\{f \in X_{m} ; \mathcal{P} f \in X_{m}\right\}$.

\section{Analysis of the subdiagonal part}

In this section, we shall consider the simplified problem corresponding to the subdiagonal part of (5),

$$
\frac{\mathrm{d} f}{\mathrm{~d} t}=\mathcal{K} f=\mathcal{G}^{-} f+\left(\mathcal{A}+\mathcal{G}^{0}+\mathcal{D}^{0}\right) f, \quad f(0)=f^{\mathrm{in}} .
$$

Denote for brevity $\mathcal{T}=\mathcal{A}+\mathcal{G}^{0}+\mathcal{D}^{0}$ and consider the operator $\left(T_{m}, D\left(T_{m}\right)\right)$ defined, as above, by $T_{m} f=\mathcal{T} f$ on $D\left(T_{m}\right)=\left\{f \in X_{m} ; \mathcal{T} f \in X_{m}\right\}$. Then, $G_{m}^{-}=\left.\mathcal{G}^{-}\right|_{D\left(T_{m}\right)}$ is a well-defined positive operator in $X_{m}$ and we can apply the substochastic semigroup theory, [8], to $\left.\mathcal{K}\right|_{D\left(T_{m}\right)}=T_{m}+G_{m}^{-}$. Let $K_{m, \max }$ denote the maximal extension of $K_{m}$; that is, $K_{m, \max } f=\mathcal{T} f+\mathcal{G}^{-} f$ on

$$
D\left(K_{m, \max }\right)=\left\{f \in X_{m} ; \sum_{n=2}^{\infty} n^{m}\left|a_{n} f_{n}+d_{n} f_{n}+g_{n} f_{n}-g_{n-1} f_{n-1}\right|<\infty\right\} .
$$


THEOREM 1. 1. If

$$
\liminf _{n \rightarrow \infty}\left(a_{n}+d_{n}-g_{n} \frac{(n+1)^{m}-n^{m}}{n^{m}}\right)>-\infty,
$$

then there is an extension $K_{m}$ of $T_{m}+G_{m}^{-}$that generates a quasicontractive (of type $\mathcal{G}(1, \omega)$ for some $\omega \in \mathbb{R}$ ) positive semigroup on $X_{m}$ and, moreover, $K_{m}=K_{m, \max }$. The resolvent $R\left(\lambda, K_{m}\right)$ for $\lambda>\omega$ is given by

$$
\left[R\left(\lambda, K_{m}\right) f\right]_{n}=\sum_{i=1}^{n} \frac{f_{i}}{\lambda+\theta_{n}} \prod_{j=i}^{n-1} \frac{g_{j}}{\lambda+\theta_{j}}, \quad n \geq 1,
$$

where $\theta_{1}=g_{1}$ and $\theta_{n}=g_{n}+d_{n}+a_{n}, n \geq 1$.

2. If there is $m^{\prime}>m$ such that ${ }^{1}$

$$
\liminf _{n \rightarrow \infty} \frac{n\left(a_{n}+d_{n}\right)}{g_{n}} \geq m^{\prime},
$$

then (10) is satisfied. Moreover, $D\left(K_{m}\right)=D\left(A_{m}\right) \cap D\left(D_{m}^{0}\right) \cap D\left(G_{m}\right)$, where $G_{m}=\left.\mathcal{G}\right|_{D\left(G_{m}\right)}$ with

$$
D\left(G_{m}\right)=\left\{f \in X_{m} ; \sum_{n=2}^{\infty}\left|g_{n} f_{n}-g_{n-1} f_{n-1}\right|<\infty\right\},
$$

and $\left(K_{m}, D\left(K_{m}\right)\right)=\overline{\left(T_{m}+G_{m}^{-}, D\left(T_{m}\right)\right)}$.

3. If (12) is satisfied, then $R\left(\lambda, K_{m}\right), \lambda>\omega$, is compact provided

$$
\liminf _{n \rightarrow \infty}\left(a_{n}+d_{n}\right)=\infty
$$

and either

$$
\sum_{n=1}^{\infty} \frac{1}{\lambda_{0}+\theta_{n}}<\infty, \quad \text { or } \quad \lim _{n \rightarrow \infty} \frac{n}{\lambda_{0}+\theta_{n}}=0
$$

for some fixed $\lambda_{0}>\omega$.

4. If

$$
\liminf _{n \rightarrow \infty} \frac{a_{n}+d_{n}}{g_{n}}>0,
$$

then $K_{m}=G_{m}^{-}+T_{m}=G_{m}^{-}+G_{m}^{0}+D_{m}^{0}+A_{m}$ and $\left(G_{K_{m}}(t)\right)_{t \geq 0}$ is an analytic semigroup. If additionally (14) is satisfied, then $\left(G_{K_{m}}(t)\right)_{t \geq 0}$ is compact.

\footnotetext{
${ }^{1}$ In (12) and everywhere below, it is assumed that the numerical coefficients like $a_{n}, d_{n}, g_{n}$, etc. take their values in the extended half line $\overline{\mathbb{R}}_{+}=[0, \infty) \cup\{+\infty\}$, where for any $a \in(0, \infty)$, we let $\frac{a}{0}=\infty$. In particular, according to this convention for a nonnegative sequence $\left(g_{n}\right)_{n \geq 0}$, with $g_{n}=0, n \geq n_{0}$, (12) holds for any finite $m^{\prime} \geq 0$.
} 
Proof. ad 1.) As in (5), we denote $\mathrm{a}_{n}=a_{n}+d_{n}, n \geq 1$. Note that by virtue of (10), $\mathrm{a}_{n}-n^{-m} g_{n}\left((n+1)^{m}-n^{m}\right) \geq \alpha>-\infty$ for $n \geq 1$. Then, if $\alpha<0$, for $f \in D\left(T_{m}\right)_{+}$, we have

$$
\begin{aligned}
& \sum_{n=1}^{\infty} n^{m}\left[\left(T_{m}+G_{m}^{-}\right) f\right]_{n}=-\sum_{n=1}^{\infty} n^{m} f_{n}\left(\mathrm{a}_{n}-g_{n} \frac{(n+1)^{m}-n^{m}}{n^{m}}\right) \\
& \quad=-\sum_{n=1}^{\infty} n^{m} f_{n}\left(\mathrm{a}_{n}-g_{n} \frac{(n+1)^{m}-n^{m}}{n^{m}}-\alpha\right)-\alpha \sum_{n=1}^{\infty} n^{m} f_{n} \\
& =-c_{1}(f)+c_{0}(f),
\end{aligned}
$$

where $c_{0}$ is a bounded functional on $X_{m}$ and $c_{1}$ is nonnegative. If $\alpha \geq 0$, we set $\alpha=0$ in the formulae above. Thus, as in [8, Proposition 9.29], there is an extension $K_{m} \supset G_{m}^{-}+T_{m}$ generating a smallest quasicontractive (with the growth rate $\omega \geq 0$ not exceeding $\left\|c_{0}\right\|$ ) positive semigroup. By [8, Theorem 6.20], $K_{m} \subset K_{m \text {,max }}$. However, in view of the two-diagonal structure of $K_{m \text {,max }}$, for $\lambda>\omega$ it is immediate that $\operatorname{Ker}\left(\lambda I-K_{m, \max }\right)=\{0\}$ and hence [8, Lemma 3.50 and Proposition 3.52] gives $K_{m}=K_{m, \max }$.

Let $\lambda>\omega$. We use the formula, see [8, Proposition 9.29],

$$
R\left(\lambda, K_{m}\right) f=\sum_{k=0}^{\infty} R\left(\lambda, T_{m}\right)\left[G_{m}^{-} R\left(\lambda, T_{m}\right)\right]^{k} f, \quad f \in X_{m}, \quad \lambda>\omega .
$$

Since $R\left(\lambda, T_{m}\right)$ is represented by the matrix $\mathcal{R}(\lambda)=\operatorname{diag}\left(\frac{1}{\lambda+\theta_{n}}\right)_{n \geq 1}$, and $G_{m}^{-}$is represented by $\mathcal{G}^{-}$, we have $\mathcal{R}(\lambda)\left[\mathcal{G}^{-} \mathcal{R}(\lambda)\right]^{k}=\left(\gamma_{i j}^{(k)}\right)_{i, j \in \mathbb{N}}$, where

$$
\gamma_{i j}^{(k)}= \begin{cases}\frac{1}{\lambda+\theta_{i}} \prod_{l=j}^{i-1} \frac{g_{l}}{\lambda+\theta_{l}}, & i \geq k+1, \quad j=i-k, \\ 0, & \text { otherwise. }\end{cases}
$$

Since the convergence in $X_{m}$ implies the coordinate-wise convergence, we see that for each $n$ the component $\left[R\left(\lambda, K_{m}\right) f\right]_{n}$ of series (18) terminates after $n$ terms and hence the resolvent is given by (11).

ad 2.) Since $c_{1}$ extends to $D\left(K_{m}\right)_{+}$and $c_{0}$ is bounded in $X_{m}$, for $f \in D\left(K_{m}\right)_{+}$, we can write

$$
\begin{aligned}
\sum_{n=1}^{\infty} n^{m}\left[\left(T_{m}+G_{m}^{-}\right) f\right]_{n} & =-\lim _{l \rightarrow \infty}\left(\sum_{n=1}^{l} n^{m} f_{n}\left(\mathrm{a}_{n}-g_{n} \frac{(n+1)^{m}-n^{m}}{n^{m}}\right)-l^{m} g_{l} f_{l}\right) \\
& =c_{0}(f)-c_{1}(f)+\lim _{l \rightarrow \infty} l^{m} g_{l} f_{l}
\end{aligned}
$$


and hence the last limit exists. Further, we have

$$
\begin{aligned}
c_{1}(f) & =\sum_{n=1}^{\infty} n^{m} f_{n}\left(-\alpha+\mathrm{a}_{n}-g_{n} \frac{(n+1)^{m}-n^{m}}{n^{m}}\right) \\
& \geq \sum_{n=1}^{\infty} n^{m} f_{n} \mathrm{a}_{n}\left(1-\frac{g_{n}}{n \mathbf{a}_{n}}\left(m+O\left(\frac{1}{n}\right)\right)\right),
\end{aligned}
$$

as $-\alpha>0$ (otherwise, we set $\alpha=0$ ). If (12) is satisfied, then (possibly adjusting $n_{0}$ from the previous part of the proof) for $n \geq n_{0}$

$$
1-\frac{g_{n}}{n \mathrm{a}_{n}}\left(m+O\left(\frac{1}{n}\right)\right) \geq 1-\frac{m}{m^{\prime}}+\frac{g_{n}}{n \mathrm{a}_{n}} O\left(\frac{1}{n}\right) \geq c^{\prime}>0
$$

on account of $m^{\prime}>m$ and $g_{n} / n \mathrm{a}_{n} \leq 1 / m^{\prime}$. Since $c_{1}$ extends to $D\left(K_{m}\right)_{+}$by monotonic limits, we argue as in [6, Theorem 2.1] that any $f \in D\left(K_{m}\right)$ is summable with the weights $\left(n^{m} \mathrm{a}_{n}\right)_{n \geq 1}$ and hence, by (12), it is also summable with the weight $\left(n^{m-1} g_{n}\right)_{n \geq 1}$. Therefore, in particular, $D\left(K_{m}\right) \subset D\left(A_{m}\right) \cap D\left(D_{m}^{0}\right)$ and hence also $D\left(K_{m}\right) \subset D\left(G_{m}\right)$ holds by the definition of $D\left(K_{\max , m}\right)$. The converse inclusion is obvious. Further, by (19), $\lim _{l \rightarrow \infty} l^{m} g_{l} f_{l}$ exists, and thus it must be 0 . Indeed, otherwise $l^{m} g_{l} f_{l}>c$ for some $c>0$ and large $l$ contradicting the summability of $\left(n^{m-1} g_{n}\right)_{n \geq 1}$. But then (19) implies that $K_{m}$ is honest; hence, $\left(K_{m}, D\left(K_{m}\right)\right)=\overline{\left(T_{m}+G_{m}^{-}, D\left(T_{m}\right)\right)}$ by [8, Corollary 6.14].

ad 3.) Though not strictly necessary, the estimates of the norm of the resolvent are instructive and used also further down. To simplify the calculations, instead of $\|\cdot\|_{[m]}$, we employ the norm $\|f\|_{*}:=\sum_{n=1}^{\infty} \frac{\Gamma(n+m)}{\Gamma(n)}\left|f_{n}\right|$ that is equivalent to $\|\cdot\|_{[m]}$ by virtue of the Gautschi inequality

$$
c_{m} n^{m} \leq \frac{\Gamma(n+m)}{\Gamma(n)}=C_{m} n^{m}, \quad n \geq 1,
$$

which holds uniformly for all $n \geq 1$, and a fixed $m>0$, with absolute constants $0<c_{m}<C_{m}$, see, e.g., [25].

Let $f \in X_{m}$ and $\lambda>\omega$. Then, changing the order of summation,

$$
\begin{aligned}
\left\|R\left(\lambda, K_{m}\right) f\right\|_{*} & \leq \sum_{i=1}^{\infty}\left|f_{i}\right| \sum_{n=i}^{\infty} \frac{\Gamma(n+m)}{\Gamma(n)} \frac{1}{\lambda+\theta_{n}} \prod_{j=i}^{n-1} \frac{g_{j}}{\lambda+\theta_{j}} \\
& =\frac{1}{\lambda-\omega} \sum_{i=1}^{\infty}\left|f_{i}\right| \sum_{n=i}^{\infty} \frac{\Gamma(n+m)}{\Gamma(n)}\left(\frac{\lambda-\omega+g_{n}}{\lambda+\theta_{n}}-\frac{g_{n}}{\lambda+\theta_{n}}\right) \prod_{j=i}^{n-1} \frac{g_{j}}{\lambda+\theta_{j}} \\
& \leq \frac{1}{\lambda-\omega} \sum_{i=1}^{\infty}\left|f_{i}\right| \sum_{n=i}^{\infty} \frac{\Gamma(n+m)}{\Gamma(n)}\left(\prod_{j=i}^{n-1} \frac{g_{j}}{\lambda+\theta_{j}}-\prod_{j=i}^{n} \frac{g_{j}}{\lambda+\theta_{j}}\right) .
\end{aligned}
$$


The internal sum can be written as

$$
\begin{aligned}
& \lim _{N \rightarrow \infty} \sum_{n=i}^{N} \frac{\Gamma(n+m)}{\Gamma(n)}\left(\prod_{j=i}^{n-1} \frac{g_{j}}{\lambda+\theta_{j}}-\prod_{j=i}^{n} \frac{g_{j}}{\lambda+\theta_{j}}\right) \\
& =\frac{\Gamma(i+m)}{\Gamma(i)}+\lim _{N \rightarrow \infty}\left(\sum_{n=i+1}^{N} \frac{\Gamma(n+m)}{\Gamma(n)} \prod_{j=i}^{n-1} \frac{g_{j}}{\lambda+\theta_{j}}-\sum_{n=i}^{N} \frac{\Gamma(n+m)}{\Gamma(n)} \prod_{j=i}^{n} \frac{g_{j}}{\lambda+\theta_{j}}\right)
\end{aligned}
$$

and then the last term can be transformed as

$$
\begin{aligned}
& \lim _{N \rightarrow \infty}\left(\sum_{n=i+1}^{N} \frac{\Gamma(n+m-1)}{\Gamma(n-1)} \prod_{j=i}^{n-1} \frac{g_{j}}{\lambda+\theta_{j}}\right. \\
& \left.\quad+m \sum_{n=i+1}^{N} \frac{\Gamma(n+m-1)}{\Gamma(n)} \prod_{j=i}^{n-1} \frac{g_{j}}{\lambda+\theta_{j}}-\sum_{n=i}^{N} \frac{\Gamma(n+m)}{\Gamma(n)} \prod_{j=i}^{n} \frac{g_{j}}{\lambda+\theta_{j}}\right) \\
& =\lim _{N \rightarrow \infty}\left(m \sum_{n=i+1}^{N} \frac{\Gamma(n+m-1)}{\Gamma(n)} \prod_{j=i}^{n-1} \frac{g_{j}}{\lambda+\theta_{j}}-\frac{\Gamma(N+m)}{\Gamma(N)} \prod_{j=i}^{N} \frac{g_{j}}{\lambda+\theta_{j}}\right) .
\end{aligned}
$$

Using (12), for sufficiently large $j$ we have

$$
\frac{g_{j}}{\lambda+\theta_{j}}=\frac{g_{j}}{\lambda+g_{j}+\left(a_{j}+d_{j}\right)} \leq \frac{j g_{j}}{\lambda j+\left(j+m^{\prime}\right) g_{j}} \leq \frac{j}{m^{\prime}+j},
$$

hence

$$
\begin{aligned}
0 & \leq \limsup _{N \rightarrow \infty} \frac{\Gamma(N+m)}{\Gamma(N)} \prod_{j=i}^{N} \frac{g_{j}}{\lambda+\theta_{j}} \leq \limsup _{N \rightarrow \infty} \frac{\Gamma(N+m)}{\Gamma(N)} \frac{\Gamma(N+1) \Gamma\left(i+m^{\prime}\right)}{\Gamma\left(N+m^{\prime}+1\right) \Gamma(i)} \\
& =\limsup _{N \rightarrow \infty} \frac{N}{N+m^{\prime}} \frac{\Gamma(N+m) \Gamma\left(i+m^{\prime}\right)}{\Gamma\left(N+m^{\prime}\right) \Gamma(i)}=0,
\end{aligned}
$$

on account of the Gautschi inequality, see (20). Thus, (22) can be estimated from the above by

$$
\begin{aligned}
& \frac{\Gamma(i+m)}{\Gamma(i)}+m \sum_{n=i+1}^{\infty} \frac{\Gamma(n+m-1)}{\Gamma(n)} \prod_{j=i}^{n-1} \frac{j}{j+m^{\prime}} \\
& =\frac{\Gamma(i+m)}{\Gamma(i)}+m \sum_{n=i+1}^{\infty} \frac{\Gamma(n+m-1) \Gamma\left(i+m^{\prime}\right)}{\Gamma(i) \Gamma\left(n+m^{\prime}\right)} \\
& =\frac{\Gamma(i+m)}{\Gamma(i)}+m \sum_{n=i+1}^{\infty} \frac{\Gamma(n+m-1)}{\Gamma(i) \Gamma(n-i)} B\left(n-i, m^{\prime}+i\right),
\end{aligned}
$$

where $B$ is the Beta function. The sum above can be computed explicitly. Indeed, using integral representation for the Beta function, the Taylor series expansion

$$
(1-t)^{-m-i}=\sum_{n=i+1}^{\infty} \frac{\Gamma(n+m-1)}{\Gamma(n-i) \Gamma(m+i)} t^{n-i-1}, \quad|t|<1,
$$


that converges pointwise in the interval $(-1,1)$, nonnegativity of the partial sums of the above series in $[0,1)$ and the Lebesgue dominated convergence theorem, we obtain

$$
\begin{aligned}
& \sum_{n=i+1}^{\infty} \frac{\Gamma(n+m-1)}{\Gamma(i) \Gamma(n-i)} \int_{0}^{1}(1-t)^{m^{\prime}+i-1} t^{n-i-1} \mathrm{~d} t \\
& =\frac{\Gamma(m+i)}{\Gamma(i)} \int_{0}^{1}\left((1-t)^{m^{\prime}+i-1} \sum_{n=i+1}^{\infty} \frac{\Gamma(n+m-1)}{\Gamma(n-i) \Gamma(m+i)} t^{n-i-1}\right) \mathrm{d} t \\
& =\frac{\Gamma(m+i)}{\Gamma(i)} \int_{0}^{1}(1-t)^{m^{\prime}-m-1} \mathrm{~d} t=\frac{\Gamma(m+i)}{\Gamma(i)} \frac{1}{m^{\prime}-m} .
\end{aligned}
$$

Substituting the above into (24) and returning to (21), we obtain

$$
\left\|R\left(\lambda, K_{m}\right) f\right\|_{*} \leq \frac{m^{\prime}}{m^{\prime}-m} \frac{1}{\lambda-\omega}\|f\|_{*} .
$$

To prove the compactness, we fix some $\lambda_{0}>\omega$ and consider the projections

$$
P_{N} f=\left(f_{1}, f_{2}, \ldots, f_{N}, 0, \ldots\right), \quad N \geq 1 .
$$

Since $P_{N} R\left(\lambda_{0}, K_{m}\right)$ is an operator with finite-dimensional range, it is compact. We consider

$$
\begin{aligned}
& \left\|P_{N-1} R\left(\lambda_{0}, K_{m}\right) f-R\left(\lambda_{0}, K_{m}\right) f\right\|_{*} \leq \sum_{n=N}^{\infty} \frac{\Gamma(n+m)}{\Gamma(n)} \sum_{i=1}^{n-1} \frac{\left|f_{i}\right|}{\lambda_{0}+\theta_{n}} \prod_{j=i}^{n-1} \frac{g_{j}}{\lambda_{0}+\theta_{j}} \\
& =\sum_{i=1}^{N-1}\left|f_{i}\right| S_{N, i}+\sum_{i=N}^{\infty}\left|f_{i}\right| S_{i+1, i},
\end{aligned}
$$

where

$$
S_{l, i}=\sum_{n=l}^{\infty} \frac{\Gamma(n+m)}{\Gamma(n)} \frac{1}{\lambda_{0}+\theta_{n}} \prod_{j=i}^{n-1} \frac{g_{j}}{\lambda_{0}+\theta_{j}} .
$$

Now,

$$
\begin{aligned}
S_{i+1, i} & =\sum_{n=i+1}^{\infty} \frac{\Gamma(n+m)}{\Gamma(n)} \frac{1}{\lambda_{0}+\theta_{n}} \prod_{j=i}^{n-1} \frac{g_{j}}{\lambda_{0}+\theta_{j}} \\
& \leq\left(\sup _{k \geq i+1} \mathrm{a}_{k}^{-1}\right) \sum_{n=i}^{\infty} \frac{\Gamma(n+m)}{\Gamma(n)} \frac{\mathrm{a}_{n}}{\lambda_{0}+\theta_{n}} \prod_{j=i}^{n-1} \frac{g_{j}}{\lambda_{0}+\theta_{j}} \\
& \leq\left(\sup _{k \geq i+1} \mathrm{a}_{k}^{-1}\right) \sum_{n=i}^{\infty} \frac{\Gamma(n+m)}{\Gamma(n)}\left(1-\frac{g_{n}}{\lambda_{0}+\theta_{n}}\right) \prod_{j=i}^{n-1} \frac{g_{j}}{\lambda_{0}+\theta_{j}}
\end{aligned}
$$




$$
\begin{aligned}
& =\left(\sup _{k \geq i+1} \mathrm{a}_{k}^{-1}\right) \sum_{n=i}^{\infty} \frac{\Gamma(n+m)}{\Gamma(n)}\left(\prod_{j=i}^{n-1} \frac{g_{j}}{\lambda_{0}+\theta_{j}}-\prod_{j=i}^{n} \frac{g_{j}}{\lambda_{0}+\theta_{j}}\right) \\
& \leq\left(\sup _{k \geq i+1} \mathrm{a}_{k}^{-1}\right) \frac{m^{\prime}}{m^{\prime}-m} \frac{\Gamma(i+m)}{\Gamma(i)}
\end{aligned}
$$

where we used the estimates for (21). Hence

$$
\begin{aligned}
\sum_{i=N}^{\infty}\left|f_{i}\right| S_{i+1, i} & \leq\left(\sup _{k \geq N+1} \mathrm{a}_{k}^{-1}\right) \frac{m^{\prime}}{m^{\prime}-m} \sum_{i=N}^{\infty}\left|f_{i}\right| \frac{\Gamma(i+m)}{\Gamma(i)} \\
& \leq\left(\sup _{k \geq N+1} \mathrm{a}_{k}^{-1}\right) \frac{m^{\prime}}{m^{\prime}-m}\|f\|_{*}
\end{aligned}
$$

and, by (14), this term tends to 0 as $N \rightarrow \infty$, uniformly on the unit ball of $X_{m}$.

Since, by (23),

$$
\prod_{j=i}^{n} \frac{g_{j}}{\lambda_{0}+\theta_{j}} \leq \prod_{j=i}^{n} \frac{j}{j+m^{\prime}} \leq \prod_{j=i}^{n} \frac{j}{j+m}=\frac{\Gamma(n) \Gamma(i+m)}{\Gamma(i) \Gamma(n+m)} \frac{n}{n+m},
$$

we have

$$
\begin{aligned}
S_{N, i} & =\sum_{n=N}^{\infty} \frac{\Gamma(n+m)}{\Gamma(n)} \frac{1}{\lambda_{0}+\theta_{n}} \prod_{j=i}^{n-1} \frac{g_{j}}{\lambda_{0}+\theta_{j}} \\
& \leq \frac{\Gamma(i+m)}{\Gamma(i)} \sum_{n=N}^{\infty} \frac{1}{\lambda_{0}+\theta_{n}} \frac{n-1}{n-1+m} \leq \frac{\Gamma(i+m)}{\Gamma(i)} \sum_{n=N}^{\infty} \frac{1}{\lambda_{0}+\theta_{n}}
\end{aligned}
$$

Hence,

$$
\sum_{i=1}^{N-1}\left|f_{i}\right| S_{N, i} \leq\left(\sum_{n=N}^{\infty} \frac{1}{\lambda_{0}+\theta_{n}}\right) \sum_{i=1}^{\infty}\left|f_{i}\right| \frac{\Gamma(i+m)}{\Gamma(i)}
$$

and, using the first option of (15) and combining the above estimate with (28), we see that

$$
\lim _{N \rightarrow \infty} P_{N-1} R\left(\lambda_{0}, K_{m}\right)=R\left(\lambda_{0}, K_{m}\right)
$$

in the uniform operator norm. Therefore, $R\left(\lambda_{0}, K_{m}\right)$ is compact.

To use the second option of (15), first we rewrite the formula for $S_{N, i}$ as

$$
\begin{aligned}
S_{N, i} & =\sum_{n=N}^{\infty} \frac{\Gamma(n+m)}{\Gamma(n)} \frac{1}{\lambda_{0}+\theta_{n}} \prod_{j=i}^{n-1} \frac{g_{j}}{\lambda_{0}+\theta_{j}} \\
& \leq \frac{\Gamma\left(i+m^{\prime}\right)}{\Gamma(i)} \sum_{n=N}^{\infty} \frac{1}{\lambda_{0}+\theta_{n}} \frac{\Gamma(n+m)}{\Gamma\left(n+m^{\prime}\right)} .
\end{aligned}
$$


Then, using again the Gautschi inequality, for large $i$ and $N>i$ we can write

$$
\frac{\Gamma\left(i+m^{\prime}\right)}{\Gamma(i)} \sum_{n=N}^{\infty} \frac{1}{\lambda_{0}+\theta_{n}} \frac{\Gamma(n+m)}{\Gamma\left(n+m^{\prime}\right)} \leq C i^{m} N^{m^{\prime}-m} \sum_{n=N}^{\infty} \frac{n^{m-m^{\prime}}}{\lambda_{0}+\theta_{n}}
$$

for some constant $C$, since $m^{\prime}-m>0$. Now, by assumption, $\frac{n^{m-m^{\prime}}}{\lambda_{0}+\theta_{n}}$ is summable (as $\lambda+\theta_{n} \geq c n$, for some $c>0$ and large values of $n$, while $m-m^{\prime}<0$ ), hence $\sum_{n=N}^{\infty} \frac{n^{m-m^{\prime}}}{\lambda_{0}+\theta_{n}}$ converges to 0 as $N \rightarrow \infty$. Since $N^{m-m^{\prime}}$ monotonically converges to 0 , we can use the Stolz-Cesáro theorem. We have

$$
\lim _{N \rightarrow \infty} N^{m^{\prime}-m} \sum_{n=N}^{\infty} \frac{n^{m-m^{\prime}}}{\lambda_{0}+\theta_{n}}=\frac{1}{m^{\prime}-m} \lim _{N \rightarrow \infty} \frac{N+1}{\lambda_{0}+\theta_{N}}=0 ;
$$

by assumption, hence we see that

$$
\sum_{i=1}^{N-1}\left|f_{i}\right| S_{N, i} \leq\left(N^{m^{\prime}-m} \sum_{n=N}^{\infty} \frac{n^{m-m^{\prime}}}{\lambda_{0}+\theta_{n}}\right) \sum_{i=1}^{\infty}\left|f_{i}\right| i^{m}
$$

and for the fixed $\lambda_{0}$ the thesis follows as above. The general case $\lambda>\omega$ follows from this and the standard resolvent identity $R\left(\lambda, K_{m}\right)=R\left(\lambda_{0}, K_{m}\right)+\left(\lambda_{0}-\right.$ $\lambda) R\left(\lambda_{0}, K_{m}\right) R\left(\lambda, K_{m}\right)$.

ad 4.) By (16), $g_{n} \leq C\left(a_{n}+d_{n}\right)$ for large $n$ and some $C>0$; hence, (12) holds and thus also the thesis of 2 . Moreover, (16) implies

$$
D\left(A_{m}\right) \cap D\left(D_{m}^{0}\right) \subset D\left(G_{m}^{0}\right) \subset D\left(T_{m}\right)
$$

and hence, by 2.), $D\left(K_{m}\right) \subset D\left(T_{m}\right)$. Since $K_{m}$ is an extension of $\left(T_{m}+G_{m}^{-}, D\left(T_{m}\right)\right)$, we see that $K_{m}=T_{m}+G_{m}^{-}$, but then we also have $K_{m}=A_{m}+D_{m}^{0}+G_{m}^{0}+G_{m}^{-}$. Further, since $\left(T_{m}, D\left(T_{m}\right)\right)$ is a diagonal operator, it generates an analytic semigroup and hence $\left(K_{m}, D\left(T_{m}\right)\right)$ also generates an analytic semigroup by the Arendt-Rhandi theorem, [2].

Now, the assumption on $g_{n}$ allows for a simpler proof of the compactness without the need for (15). By virtue of the above and [8, Theorem 4.3], $I-G_{m}^{-} R\left(\lambda, T_{m}\right)$ is invertible and

$$
R\left(\lambda, T_{m}+G_{m}^{-}\right)=R\left(\lambda, T_{m}\right)\left[I-G_{m}^{-} R\left(\lambda, T_{m}\right)\right]^{-1} .
$$

In view of the last identity, it suffices to show that $R\left(\lambda, T_{m}\right)$ is compact for some $\lambda>0$. However, the latter is immediate, for by virtue of $(14), R\left(\lambda, T_{m}\right)$ is the uniform limit of finite rank operators.

REMARK 1. We note that (16) also allows to apply the Miyadera perturbation theorem, see, e.g., [23, Theorem III.3.16]. Indeed, if (16) is satisfied, then we can find $n_{0}$ such that for $n \geq n_{0}+1$

$$
\frac{(n+1)^{m} g_{n}}{n^{m}\left(g_{n}+\mathrm{a}_{n}\right)} \leq q<1
$$


and then $\omega>0$ such that

$$
\max _{1 \leq n \leq n_{0}} \frac{(n+1)^{m} g_{n}}{n^{m}\left(g_{n}+\mathrm{a}_{n}+\omega\right)} \leq q<1 .
$$

Since the generation for $T_{m}+G_{m}^{-}$is equivalent to that for $T_{m}+G_{m}^{-}-\omega I$, [8, Lemma 4.15], the Miyadera condition for $T_{m}+G_{m}^{-}-\omega I$ and $f \in D\left(T_{m}\right)_{+}$reads

$$
\begin{aligned}
\int_{0}^{\delta}\left\|G_{m}^{-} G_{T_{m}}(t) f\right\|_{[m]} \mathrm{d} t & =\sum_{n=1}^{\infty} \frac{(n+1)^{m} g_{n}\left(1-e^{-\left(g_{n}+\mathrm{a}_{n}+\omega\right) \delta}\right)}{n^{m}\left(g_{n}+\mathrm{a}_{n}+\omega\right)} n^{m} f_{n} \\
& \leq \sum_{n=1}^{n_{0}} \frac{(n+1)^{m} g_{n}}{n^{m}\left(g_{n}+\mathrm{a}_{n}+\omega\right)} n^{m} f_{n}+\sum_{n=n_{0}+1}^{\infty} \frac{(n+1)^{m} g_{n}}{n^{m}\left(g_{n}+\mathrm{a}_{n}\right)} n^{m} f_{n} \\
& \leq q\|f\|_{[m]} .
\end{aligned}
$$

At the same time, if $\mathrm{a}_{n} / g_{n} \rightarrow 0$ as $n \rightarrow \infty$, then $g_{n} /\left(\mathrm{a}_{n}+g_{n}\right) \rightarrow 1$ and the above estimate is not available.

\section{Growth-fragmentation equation}

We introduce the following notation, see [6],

$$
\Delta_{n}^{(m)}:=n^{m}-b_{n}^{(m)}:=n^{m}-\sum_{k=1}^{n-1} k^{m} b_{k, n}, \quad n \geq 2, \quad m \geq 0 .
$$

Then, for $n \geq 2$,

$$
\Delta_{n}^{(0)}=1-b_{1}^{0} \leq 0, \quad \Delta_{n}^{(1)}=0, \quad \Delta_{n}^{(m)} \geq 0, \quad m>1 .
$$

Further, let us recall the notation $\theta_{1}=g_{1}$ and $\theta_{n}=a_{n}+g_{n}+d_{n}, n \geq 2$.

THEOREM 2. 1. Let (12) be satisfied. If for some $m^{\prime}>m>1$

$$
\liminf _{n \rightarrow \infty} \frac{a_{n}}{\mathrm{a}_{n}} \frac{\Delta_{n}^{(m)}}{n^{m}}>\frac{m}{m^{\prime}}
$$

holds, where as before $\mathrm{a}_{n}=a_{n}+d_{n}$, then

$$
\begin{aligned}
\left(Y_{m}, D\left(K_{m}\right)\right) & =\frac{\left(K_{m}+D_{m}^{+}+B_{m}, D\left(K_{m}\right)\right)}{\left(T_{m}+G_{m}^{-}+D_{m}^{+}+B_{m}, D\left(T_{m}\right)\right)}
\end{aligned}
$$

generates a positive semigroup in $X_{m}$. If additionally (14) and (15) are satisfied, $R\left(\lambda, Y_{m}\right)$ is compact for sufficiently large $\lambda$. 
2. Iffor some $m>1$

$$
\liminf _{n \rightarrow \infty} \frac{a_{n}}{\theta_{n}} \frac{\Delta_{n}^{(m)}}{n^{m}}>0
$$

holds, then

$$
\left(U_{m}, D\left(T_{m}\right)\right)=\left(A_{m}+G_{m}+D_{m}+B_{m}, D\left(T_{m}\right)\right)=\left(Y_{m}, D\left(Y_{m}\right)\right),
$$

where $D\left(T_{m}\right)=D\left(A_{m}+G_{m}^{0}+D_{m}^{0}\right)$, generates a positive, analytic semigroup in $X_{k}$ for any $k \geq m$.

Proof. ad 1.) Using the same ideas as in the proof of Theorem 1, part 1, for the full operator with the aid of $f \in D\left(K_{m}\right)_{+} \subset D\left(A_{m}\right)_{+} \cap D\left(D_{m}^{0}\right)_{+}$and (13), we obtain

$$
\sum_{n=1}^{\infty} n^{m}\left[\left(K_{m}+D_{m}^{+}+B_{m}\right) f\right]_{n}=\sum_{n=1}^{\infty} n^{m}\left[\left(A_{m}+D_{m}+B_{m}\right) f\right]_{n}+\sum_{n=1}^{\infty} n^{m}\left[G_{m} f\right]_{n} .
$$

Now, using the convention that $g_{0} f_{0}=0$,

$$
\begin{aligned}
& \sum_{n=1}^{\infty} n^{m}\left[G_{m} f\right]_{n}=\lim _{l \rightarrow \infty} \sum_{n=1}^{l} n^{m}\left(g_{n-1} f_{n-1}-g_{n} f_{n}\right) \\
& \quad=\lim _{l \rightarrow \infty}\left(\sum_{n=1}^{l}\left((n+1)^{m}-n^{m}\right) g_{n} f_{n}-(l+1)^{m} g_{l+1} f_{l+1}\right) \\
& \quad=\sum_{n=1}^{\infty}\left((n+1)^{m}-n^{m}\right) g_{n} f_{n},
\end{aligned}
$$

by the proof of Theorem 1, part 2. Hence,

$$
\begin{aligned}
& \sum_{n=1}^{\infty} n^{m}\left[\left(K_{m}+D_{m}^{+}+B_{m}\right) f\right]_{n}=\sum_{n=1}^{\infty} \mathrm{a}_{n} n^{m} f_{n}\left(\left(\left(1+\frac{1}{n}\right)^{m}-1\right) \frac{g_{n}}{\mathrm{a}_{n}}\right. \\
& \left.\quad-\frac{a_{n}}{\mathrm{a}_{n}}\left(1-\frac{1}{n^{m}} \sum_{k=1}^{n-1} k^{m} b_{k, n}\right)-\frac{d_{n}}{\mathrm{a}_{n}}\left(1-\left(1-\frac{1}{n}\right)^{m}\right)\right) \\
& =:-\sum_{n=1}^{\infty} \Lambda_{n} \mathrm{a}_{n} n^{m} f_{n} .
\end{aligned}
$$

Thus, if $\Lambda_{n} \geq 0$ for large $n$, then there is an extension $\left(Y_{m}, D\left(Y_{m}\right)\right)$ of $\left(K_{m}+D_{m}^{+}+\right.$ $\left.B_{m}, D\left(A_{m}\right) \cap D\left(D_{m}^{0}\right) \cap D\left(G_{m}\right)\right)$ generating a positive semigroup. Since

$$
\Lambda_{n}=\frac{a_{n}}{\mathrm{a}_{n}} \frac{\Delta_{n}^{(m)}}{n^{m}}+\frac{d_{n}}{\mathrm{a}_{n}} O\left(\frac{1}{n}\right)-\frac{g_{n}}{n \mathrm{a}_{n}}\left(m+O\left(\frac{1}{n}\right)\right),
$$

where both $\frac{d_{n}}{\mathrm{a}_{n}} O\left(\frac{1}{n}\right)$ and $\frac{g_{n}}{n \mathrm{a}_{n}} O\left(\frac{1}{n}\right)$ converge to zero due to the boundedness of $d_{n} / \mathrm{a}_{n}$ and $\frac{g_{n}}{n \mathbf{a}_{n}} \leq \frac{1}{m^{\prime}}+\varepsilon_{n}$, with $\varepsilon_{n} \rightarrow 0^{+}$as $n \rightarrow \infty$ (see (12)), we have $\Lambda_{n} \geq \Lambda>$ 
0 for some $\Lambda$ and large $n$, provided (31) is satisfied. We observe that, in view of (29) and the trivial inequality $\frac{a_{n}}{\mathrm{a}_{n}} \leq 1, \Lambda \leq 1$ so that $1<m<m^{\prime}$ is a necessary condition for (31) to hold. Hence, if (31) holds, $D\left(Y_{m}\right) \subset D\left(A_{m}\right) \cap D\left(D_{m}^{0}\right)$. Then, since $D\left(B_{m}\right), D\left(D_{m}^{+}\right) \subset D\left(A_{m}\right) \cap D\left(D_{m}^{0}\right)$ and $Y_{m}$ is a restriction of the maximal operator, $D\left(Y_{m}\right) \subset D\left(G_{m}\right)$ and hence the first part of (32) is proved. To prove the second part, we note that $\left(K_{m}, D\left(K_{m}\right)\right)=\overline{\left(T_{m}+G_{m}^{-}, D\left(T_{m}\right)\right)}$. Since $K_{m}+D_{m}^{+}+B_{m}$ is the generator, it is closed and thus

$$
\begin{aligned}
\overline{\left(T_{m}+G_{m}^{-}+D_{m}^{+}+B_{m}, D\left(T_{m}\right)\right)} & \subset \overline{\left(K_{m}+D_{m}^{+}+B_{m}, D\left(K_{m}\right)\right)} \\
& =\left(K_{m}+D_{m}^{+}+B_{m}, D\left(K_{m}\right)\right) .
\end{aligned}
$$

On the other hand, $D\left(K_{m}\right) \subset D\left(D_{m}^{0}\right) \cap D\left(A_{m}\right)=D\left(D_{m}^{+}\right) \cap D\left(B_{m}\right)$, hence $D_{m}^{+}+B_{m}$ is $K_{m}$-bounded by [8, Lemma 4.1 and Theorem 2.65]. Let $f \in D\left(K_{m}\right)$. Then, $f=$ $\lim _{n \rightarrow \infty} f_{n}$ with $f_{n} \in D\left(T_{m}\right)$ and $\lim _{n \rightarrow \infty} K_{m} f_{n}=\lim _{n \rightarrow \infty}\left(T_{m}+G_{m}^{-}\right) f_{n}=K_{m} f$. By $K_{m}$-boundedness, $\left(\left(D_{m}^{+}+B_{m}\right) f_{n}\right)_{n \in \mathbb{N}}$ converges. By (36), $T_{m}+G_{m}^{-}+D_{m}^{+}+B_{m}$ is closable and hence

$$
\begin{aligned}
K_{m} f+D_{m}^{+} f+B_{m} f & =\lim _{n \rightarrow \infty}\left(T_{m}+G_{m}^{-}+D_{m}^{+}+B_{m}\right) f_{n} \\
& =\overline{\left(T_{m}+G_{m}^{-}+D_{m}^{+}+B_{m}\right)} f .
\end{aligned}
$$

Thus,

$$
K_{m}+D_{m}^{+}+B_{m} \subset \overline{T_{m}+G_{m}^{-}+D_{m}^{+}+B_{m}}
$$

and (32) follows.

The compactness of $R\left(\lambda, Y_{m}\right)$ follows from

$$
R\left(\lambda, Y_{m}\right)=R\left(\lambda, K_{m}\right)\left[I-\left(B_{m}+D_{m}^{+}\right) R\left(\lambda, K_{m}\right)\right]^{-1},
$$

where the second term on the right-hand side is a bounded operator by $D\left(B_{m}+D_{m}^{+}\right)=$ $D\left(A_{m}\right) \cap D\left(D_{m}^{0}\right) \supset D\left(K_{m}\right)$. Thus, the proof of the compactness of $R\left(\lambda, Y_{m}\right)$ follows as in item 4.) of Theorem 1.

ad 2.) By [6, Theorem 2.1], if (33) holds for some $m_{0}$, then it holds for any $m \geq m_{0}$. Hence, we can fix an $m$ for which (33) holds. Then, for $f \in D\left(T_{m}\right)=D\left(A_{m}+G_{m}^{0}+\right.$ $\left.D_{m}^{0}\right)=D\left(A_{m}\right) \cap D\left(G_{m}^{0}\right) \cap D\left(D_{m}^{0}\right)$, we obtain

$$
\begin{aligned}
& \sum_{n=1}^{\infty} n^{m}\left[\left(G_{m}+D_{m}+A_{m}+B_{m}\right) f\right]_{n}=\sum_{n=1}^{\infty} \theta_{n} n^{m} f_{n}\left(\left(\left(1+\frac{1}{n}\right)^{m}-1\right) \frac{g_{n}}{\theta_{n}}\right. \\
& \left.\quad-\frac{a_{n}}{\theta_{n}}\left(1-\frac{1}{n^{m}} \sum_{k=1}^{n-1} k^{m} b_{k, n}\right)-\frac{d_{n}}{\theta_{n}}\left(1-\left(1-\frac{1}{n}\right)^{m}\right)\right) \\
& =:-\sum_{n=1}^{\infty} \theta_{n} n^{m} f_{n} \Theta_{n} .
\end{aligned}
$$


Then, we proceed as above. Since

$$
\Theta_{n}=\frac{a_{n}}{\theta_{n}} \frac{\Delta_{n}^{(m)}}{n^{m}}+\frac{d_{n}}{\theta_{n}} O\left(\frac{1}{n}\right)-\frac{g_{n}}{\theta_{n}} O\left(\frac{1}{n}\right),
$$

where the terms $\frac{g_{n}}{\theta_{n}} O\left(\frac{1}{n}\right)$ and $\frac{d_{n}}{\theta_{n}} O\left(\frac{1}{n}\right)$ converge to zero due to the boundedness of $g_{n} / \theta_{n}$ and $d_{n} / \theta_{n}, \Theta_{n} \geq c>0$ for large $n$ if and only if (33) is satisfied. Hence, $\left(U_{m}, D\left(T_{m}\right)\right):=\left(G_{m}+D_{m}+A_{m}+B_{m}, D\left(A_{m}+G_{m}^{0}+D_{m}^{0}\right)\right)$ generates an analytic semigroup as the positive perturbation of the diagonal operator $\left(T_{m}, D\left(T_{m}\right)\right)$. However, by the closedness,

$$
\begin{aligned}
& \left(A_{m}+G_{m}+D_{m}+B_{m}, D\left(A_{m}+G_{m}^{0}+D_{m}^{0}\right)\right) \\
& \quad=\left(T_{m}+G_{m}^{-}+D_{m}^{+}+B_{m}, D\left(T_{m}\right)\right) \\
& \quad=\overline{\left(T_{m}+G_{m}^{-}+D_{m}^{+}+B_{m}, D\left(T_{m}\right)\right)}=\left(Y_{m}, D\left(Y_{m}\right)\right) .
\end{aligned}
$$

REMARK 2. We note that (33) implies that both $\frac{a_{n}}{\theta_{n}}$ and $\frac{\Delta_{n}^{(m)}}{n^{m}}$ must be bounded away from 0 and thus, in particular, (16) is satisfied so that $\left(G_{K_{m}}(t)\right)_{t \geq 0}$ and the pure fragmentation semigroup is analytic and, provided (14) holds, compact in its own right.

\section{Asynchronous exponential growth}

PROPOSITION 1. If Assumptions (14) and (33) are satisfied, then the semigroup $\left(G_{U_{m}}(t)\right)_{t \geq 0}$ is analytic and compact.

Proof. By (34), $\left(G_{U_{m}}(t)\right)_{t \geq 0}$ can be considered to be generated as the perturbation $U_{m}=T_{m}+\left(G_{m}^{-}+D_{m}^{+}+B_{m}\right)$ so, as in the proof of Theorem 1.4, $R\left(\lambda, T_{m}\right) X_{m} \subset$ $D\left(U_{m}\right)$ implies

$$
R\left(\lambda, U_{m}\right)=R\left(\lambda, T_{m}\right)\left(I-\left(G_{m}^{-}+D_{m}^{+}+B_{m}\right) R\left(\lambda, T_{m}\right)\right)^{-1},
$$

where, by

$$
\left(\lambda I-T_{m}\right) R\left(\lambda, U_{m}\right)=\left(I-\left(G_{m}^{-}+D_{m}^{+}+B_{m}\right) R\left(\lambda, T_{m}\right)\right)^{-1},
$$

the operator $\left(I-\left(G_{m}^{-}+D_{m}^{+}+B_{m}\right) R\left(\lambda, T_{m}\right)\right)^{-1}$ is bounded. Hence, $R\left(\lambda, U_{m}\right)$ is compact, provided $R\left(\lambda, T_{m}\right)$ is compact and that was proved in Theorem 1.4. Since $\left(G_{U_{m}}(t)\right)_{t \geq 0}$ is analytic, its compactness follows from [23, Theorem II.4.29].

PROPOSITION 2. The semigroup $\left(G_{U_{m}}(t)\right)_{t \geq 0}$ is irreducible if and only if $g_{n}>0$ for all $n \geq 1$. 
Proof. Necessity. If $g_{n_{0}}=0$ for some $n_{0} \geq 1$, then the closed $n_{0}$-dimensional subspace of $X_{m}$, spanned by the canonical vectors $e_{n}=\left(\delta_{i, n}\right)_{i \geq 1}, 1 \leq n \leq n_{0}$ is invariant under the action of the generator $\left(U_{m}, D\left(T_{m}\right)\right)$ and hence the semigroup $\left(G_{U_{m}}(t)\right)_{t \geq 0}$ is not irreducible.

Sufficiency. By [15, Proposition 7.6] it suffices to show that $R\left(\lambda, U_{m}\right) e_{n}>0$ for all $n \geq 1$ and for some fixed $\lambda>s\left(U_{m}\right)$. To simplify the calculations, we use the representation

$$
\left(U_{m}, D\left(T_{m}\right)\right)=\left(K_{m}+D_{m}^{+}+B_{m}, D\left(T_{m}\right)\right)=:\left(K_{m}+\mathrm{B}_{m}, D\left(T_{m}\right)\right)
$$

(see (34), corresponding to (5) and (6)), which combined with the resolvent formula from [8, Proposition 9.29] (compare (18)), gives

$$
R\left(\lambda, U_{m}\right) f=\sum_{k=0}^{\infty} R\left(\lambda, K_{m}\right)\left[\mathrm{B}_{m} R\left(\lambda, K_{m}\right)\right]^{k} f, \quad f \in X_{m}, \quad \lambda>s\left(U_{m}\right) .
$$

Let $n_{0} \geq 1$ be fixed. From (37) and (11), we infer

$$
\begin{aligned}
& {\left[R\left(\lambda, U_{m}\right) e_{n_{0}}\right]_{n} \geq\left[R\left(\lambda, K_{m}\right)\left(I+\mathrm{B}_{m} R\left(\lambda, K_{m}\right)\right) e_{n_{0}}\right]_{n}} \\
& \quad=\sum_{i=1}^{n} \frac{1}{\lambda+\theta_{n}} \prod_{k=i}^{n-1} \frac{g_{k}}{\lambda+\theta_{k}}\left(\delta_{i, n_{0}}+\sum_{j=i+1}^{\infty} \mathrm{a}_{j} \mathrm{~b}_{i, j}\left(\sum_{s=1}^{j} \frac{\delta_{s, n_{0}}}{\lambda+\theta_{j}} \prod_{l=s}^{j-1} \frac{g_{l}}{\lambda+\theta_{l}}\right)\right) .
\end{aligned}
$$

In view of our assumptions, the last formula indicates that $\left[R\left(\lambda, U_{m}\right) e_{n_{0}}\right]_{n}>0$, provided $n \geq n_{0}$. Moreover, for $n_{0}>1$,

$$
\begin{aligned}
& \left.\left[R\left(\lambda, K_{m}\right) \mathrm{B}_{m} R\left(\lambda, K_{m}\right)\right) e_{n_{0}}\right]_{n_{0}-1} \\
& \quad=\sum_{i=1}^{n_{0}-1} \frac{1}{\lambda+\theta_{n_{0}-1}} \prod_{k=i}^{n_{0}-2} \frac{g_{k}}{\lambda+\theta_{k}}\left(\sum_{j=i+1}^{\infty} \mathrm{a}_{j} \mathrm{~b}_{i, j} \frac{1}{\lambda+\theta_{j}} \prod_{l=n_{0}}^{j-1} \frac{g_{l}}{\lambda+\theta_{l}}\right)>0
\end{aligned}
$$

for, if not, then

$$
\mathrm{b}_{i, n_{0}}=0, \quad 1 \leq i \leq n_{0}-1
$$

as all other terms and multipliers are positive. This contradicts (7) that requires

$$
\sum_{i=1}^{n_{0}-1} i \mathrm{~b}_{i, n_{0}}=n_{0}-\frac{d_{n_{0}}}{a_{n_{0}}+d_{n_{0}}}>0, \quad n_{0}>1
$$

Thus, if $n_{0}=2$, then $\left[R\left(\lambda, U_{m}\right) e_{n_{0}}\right]_{n}>0$ for $n \geq 1$. If $n_{0}>2$, then we consider the third term in (37), evaluated at $e_{n_{0}}$,

$$
\begin{aligned}
R\left(\lambda, K_{m}\right)\left[\mathrm{B}_{m} R\left(\lambda, K_{m}\right)\right]^{2} e_{n_{0}} & =R\left(\lambda, K_{m}\right) \mathrm{B}_{m}\left[R\left(\lambda, K_{m}\right) \mathrm{B}_{m} R\left(\lambda, K_{m}\right) e_{n_{0}}\right] \\
& =R\left(\lambda, K_{m}\right) \mathrm{B}_{m} \Phi
\end{aligned}
$$


where $\Phi=\left(\phi_{n}\right)_{n \geq 1}$ is a sequence with $\phi_{n}>0$ for $n \geq n_{0}-1$. Since in the proof of (38) we only used the fact that $R\left(\lambda, K_{m}\right) \mathrm{B}_{m}$ acted on a sequence with a positive $n_{0}$ entry, we see in the same way that

$$
\left[R\left(\lambda, K_{m}\right)\left[\mathrm{B}_{m} R\left(\lambda, K_{m}\right)\right]^{2} e_{n_{0}}\right]_{n_{0}-2}>0 .
$$

Repeating the argument, for $n_{0}>k$ we have

$$
\left[R\left(\lambda, K_{m}\right)\left[B_{m} R\left(\lambda, K_{m}\right]^{k} e_{n_{0}}\right]_{n_{0}-k}>0, \quad 1 \leq k \leq n_{0}-1\right.
$$

and hence $R\left(\lambda, U_{m}\right) e_{n_{0}}>0$. Since any $f \in X_{m,+}, f \neq 0$, is bounded from below by a finite linear combination of elements from $\left\{e_{n}\right\}_{n \geq 1}$, we conclude that $R\left(\lambda, U_{m}\right)$, and hence $\left(G_{U_{m}}(t)\right)_{t \geq 0}$, are irreducible.

Thus [24, Theorem VI.3.5] yields the following result.

THEOREM 3. Assume that $g_{n}>0, n \geq 1$ and (14) and (33) are satisfied. Then, there exist a strictly positive $e \in X_{m}$, a strictly positive $h \in X_{m}^{*}, M \geq 1$ and $\epsilon>0$ such that for any $f^{\text {in }} \in X_{n}$ and $t \geq 0$

$$
\left\|e^{-s\left(U_{m}\right) t} G_{U_{m}}(t) f^{\mathrm{in}}-\left\langle h, f^{\mathrm{in}}\right\rangle e\right\|_{[m]} \leq M e^{-\epsilon t} .
$$

\section{Examples}

EXAMPLE 1. To illustrate the above result, consider the growth-fragmentation problem

$$
\begin{aligned}
\frac{\mathrm{d} f_{1}}{\mathrm{~d} t} & =-g_{1} f_{1}+\sum_{i=2}^{\infty} a_{i} b_{1, i} f_{i}, \\
\frac{\mathrm{d} f_{n}}{\mathrm{~d} t} & =g_{n-1} f_{n-1}-\left(a_{n}+g_{n}\right) f_{n}, \quad n \geq 2 \\
f_{n}(0) & =f_{n}^{\text {in }}, \quad n \geq 1,
\end{aligned}
$$

where

$$
b_{n, i}= \begin{cases}i & \text { for } n=1 \\ 0 & \text { otherwise }\end{cases}
$$

that is, any particle breaks down into monomers. Since $d_{n}=0$ for all $n$, we take any unbounded $\left(a_{n}\right)_{n \in \mathbb{N}}$ and $\left(g_{n}\right)_{n \in \mathbb{N}}$ satisfying (14) and

$$
\gamma a_{n} \leq g_{n} \leq g a_{n}, \quad n \geq 2
$$


for some $\gamma \leq g$. We note that in this settings $\Delta_{n}^{(m)}=n^{m}-n$ and (33) holds for any $m>1$. Hence, the semigroup $\left(G_{U_{m}}(t)\right)_{t \geq 0}$ that solves (40) is analytic and compact in $X_{m}$ for any $m>1$ and Theorem 3 holds. Moreover,

$$
\begin{aligned}
& \lambda f_{1}=-g_{1} f_{1}+\sum_{i=2}^{\infty} a_{i} b_{1, i} f_{i}, \\
& \lambda f_{n}=g_{n-1} f_{n-1}-\left(a_{n}+g_{n}\right) f_{n}, \quad n \geq 2
\end{aligned}
$$

can be explicitly solved. Indeed, let $\lambda \geq 0$ and, starting from the second equation, we get

$$
f_{n, \lambda}=\frac{g_{1} f_{1, \lambda}}{\lambda+g_{n}+a_{n}} \prod_{j=2}^{n-1} \frac{g_{j}}{\lambda+g_{j}+a_{j}}, \quad n \geq 2
$$

and

$$
\sum_{n=2}^{\infty} a_{n} b_{1, n} f_{n, \lambda}=g_{1} f_{1, \lambda} \sum_{n=2}^{\infty} \frac{a_{n} n}{\lambda+a_{n}+g_{n}} \prod_{j=2}^{n-1} \frac{g_{j}}{\lambda+g_{j}+a_{j}} .
$$

Now, by (41) (see (16)), $\frac{g_{j}}{\lambda+g_{j}+a_{j}} \leq c=\frac{g}{1+g}<1$ and thus,

$$
\sum_{n=2}^{\infty} \frac{a_{n} n}{\lambda+a_{n}+g_{n}} \prod_{j=2}^{n-1} \frac{g_{j}}{\lambda+g_{j}+a_{j}} \leq \sum_{n=2}^{\infty} n c^{n-2}<\infty .
$$

Hence, after dividing by $g_{1} f_{1, \lambda} \neq 0$, the first equation in (42) takes the form

$$
\psi(\lambda):=\frac{\lambda+g_{1}}{g_{1}}=\sum_{n=2}^{\infty} \frac{a_{n} n}{\lambda+a_{n}+g_{n}} \prod_{j=2}^{n-1} \frac{g_{j}}{\lambda+g_{j}+a_{j}}=: \phi(\lambda)
$$

and the eigenvalue problem reduces to the algebraic equation $\psi(\lambda)=\phi(\lambda)$. By (43), the series defining $\phi$ is uniformly convergent on $[0, \infty)$; hence, $\phi$ is continuous there and

$$
\phi(0)=\sum_{n=2}^{\infty} \frac{a_{n} n}{g_{n}} \prod_{j=2}^{n} \frac{g_{j}}{g_{j}+a_{j}} .
$$

Using (41), we have, for $q=\frac{\gamma}{1+\gamma}$,

$\phi(0) \geq \frac{1}{g} \sum_{n=2}^{\infty} n q^{n-1}=\frac{1}{g} \frac{\mathrm{d}}{\mathrm{d} q} \sum_{n=2}^{\infty} q^{n}=\frac{1}{g} \frac{\mathrm{d}}{\mathrm{d} q} \frac{q^{2}}{1-q}=\frac{1}{g} \frac{2 q-q^{2}}{(1-q)^{2}}=\frac{1}{g}\left(\frac{1}{(1-q)^{2}}-1\right) ;$

that is,

$$
\phi(0) \geq \frac{(\gamma+1)^{2}-1}{g}>1
$$


provided

$$
g+1<(\gamma+1)^{2} \leq(g+1)^{2},
$$

where the second inequality follows from $\gamma \leq g$, implied by (41). We see that, in particular, if $\gamma=g$, that is, $g_{n}=g a_{n}$, then (44) is satisfied. Also, $\lim _{\lambda \rightarrow \infty} \phi(\lambda)=0$. On the other hand, $\psi(0)=1$ and $\lim _{\lambda \rightarrow \infty} \psi(\lambda)=+\infty$. Since $\phi$ is decreasing and $\psi$ is increasing, there is exactly one $\lambda_{0}>0$ for which (42) has a solution (with arbitrary $f_{1}$ that can be set to 1$)$. Moreover, we see that

$$
\sum_{n=1}^{\infty} a_{n} n^{m} f_{n, \lambda}=g_{1} \sum_{n=2}^{\infty} \frac{a_{n} n^{m}}{\lambda+a_{n}+g_{n}} \prod_{j=2}^{n-1} \frac{g_{j}}{\lambda+g_{j}+a_{j}} \leq g_{1} \sum_{n=2}^{\infty} n^{m} c^{n-2}<\infty,
$$

and thus $f_{\lambda_{0}}=\left(f_{n, \lambda_{0}}\right)_{n \in \mathbb{N}}$ is the Perron eigenvector of the generator $U_{m}$.

EXAMPLE 2. The dominant eigenvalue $\lambda_{0}$ can be explicitly found in certain cases. Let us consider general problem (1) with $g_{n}=r n, d_{n}=0$ for all $n \in \mathbb{N}$ and some $r>0$ and with other coefficients satisfying the assumptions of Theorem 3. Let $f_{\lambda}=$ $\left(f_{n, \lambda}\right)_{n \in \mathbb{N}} \in D\left(U_{m}\right)$ satisfy

$$
\begin{aligned}
& \lambda f_{1, \lambda}=-r f_{1, \lambda}+\sum_{i=2}^{\infty} a_{i} b_{1, i} f_{i, \lambda}, \\
& \lambda f_{n, \lambda}=r(n-1) f_{n-1, \lambda}-\left(a_{n}+r n\right) f_{n, \lambda}+\sum_{i=n+1}^{\infty} a_{i} b_{n, i} f_{i, \lambda}, \quad n \geq 2 .
\end{aligned}
$$

Multiplying the $n$th equation by $n$ and summing them, we obtain

$$
\lambda \sum_{n=1}^{\infty} n f_{n, \lambda}=r \sum_{n=1}^{\infty} n f_{n, \lambda}
$$

The above is satisfied if either $\lambda=r$ or $\sum_{n=1}^{\infty} n f_{n, \lambda}=0$. Since we know that the Perron eigenvector must be positive, we obtain that $\lambda_{0}=r$ is the Perron eigenvalue. As a by-product, we see that any eigenvector $f_{\lambda}$ belonging to an eigenvalue $\lambda \neq r$ must satisfy $\sum_{n=1}^{\infty} n f_{n, \lambda}=0$.

To conclude, let us consider the transposed matrix

$$
\mathcal{U}^{T}=\left(\begin{array}{cccccc}
-g_{1} & g_{1} & 0 & 0 & 0 & \ldots \\
a_{2} b_{1,2} & -\left(g_{2}+a_{2}\right) & g_{2} & 0 & 0 & \ldots \\
a_{3} b_{1,3} & a_{3} b_{2,3} & -g_{3}+a_{3} & g_{3} & 0 & \ldots \\
\vdots & \vdots & \vdots & \vdots & \vdots & \vdots \\
a_{n} b_{1, n} & a_{n} b_{2, n} & \ldots & -\left(g_{n}+a_{n}\right) & g_{n} & \ldots \\
\vdots & \vdots & \vdots & \vdots & \vdots & \vdots
\end{array}\right) .
$$

Let $U_{m}^{*}$ be the adjoint to $U_{m}$ acting in $X_{m}^{*}=\left\{\left(v_{n}\right)_{n \in \mathbb{N}} ; \sup _{n \in \mathbb{N}} n^{-m}\left|v_{n}\right|<\infty\right\}$ and let $f^{*} \in D\left(U_{m}^{*}\right)$. Then, by definition

$$
\left\langle U_{m}^{*} f^{*}, f\right\rangle=\left\langle f^{*}, U_{m} f\right\rangle, \quad f \in D\left(U_{m}\right) .
$$


Taking $f=\left(\delta_{n, N}\right)_{n \in \mathbb{N}}$, we see that $\left[U_{m}^{*} f^{*}\right]_{N}=\left\langle f^{*}, U_{m} f\right\rangle=\sum_{n=1}^{N-1} f_{n}^{*} a_{N} b_{n, N}-\left(g_{N}+a_{N}\right) f_{N}^{*}+g_{N} f_{N+1}^{*}=\left[\mathcal{U}^{T} f^{*}\right]_{N}$

hence $U_{m}^{*}$ is a restriction of $\mathcal{U}^{T}$ to $D\left(U_{m}^{*}\right) \subset D\left(U_{m, \max }^{*}\right)=\left\{f \in X_{m}^{*}: \mathcal{U}^{T} f \in X_{m}^{*}\right\}$. On the other hand, let $f^{*} \in D\left(U_{m, \max }^{*}\right), f \in D\left(U_{m}\right)$. Then, since $D\left(U_{m}\right)$ is a weighted $l^{1}$ space, $\bigcup_{N=1}^{\infty} P_{N} D\left(U_{m}\right)$, where $P_{N}$ is the projection defined in (26), is a core for $U_{m}$. Using the fact that $U_{m} P_{N} D\left(U_{m}\right)$ is finite dimensional, for each $N$

$$
\left\langle\mathcal{U}^{T} f^{*}, P_{N} f\right\rangle=\left\langle f^{*}, U_{m} P_{N} f\right\rangle=\left\langle U_{m}^{*} f^{*}, P_{N} f\right\rangle
$$

and hence, passing to the limit with $N \rightarrow \infty, f^{*} \in D\left(U_{m}^{*}\right)$. Thus, $U_{m}^{*}=\mathcal{U}^{T}$ with $D\left(U_{m}^{*}\right)=D\left(U_{m, \max }^{*}\right)$.

Using the assumption that $g_{n}=r n$, we see that $h=(1,2, \ldots, n, \ldots) \in D\left(U_{m}^{*}\right)$ for any $m \geq 1$ and

$$
U_{m}^{*} h=r h .
$$

Thus, by Theorem 3,

$$
U_{m}(t) f^{\text {in }}=e^{r t}\left(\sum_{n=1}^{\infty} n f_{n}^{\text {in }}\right) e+O\left(e^{r^{\prime} t}\right)
$$

for some $r^{\prime}<r$, where $e$ is the Perron eigenvector with unit mass; that is $e=$ $f_{\lambda_{0}} / \sum_{n=1}^{\infty} n f_{n, \lambda_{0}}$.

To illustrate the formulas derived in the last two examples, we let $m=2, r=1$, $a_{n}=2 n, f_{n}^{\text {in }}=\delta_{n, 10} 10$ and integrate (40) numerically in the time interval $t \in[0,20]$. As evident from Fig. 1, the solution $f(t)$ very quickly settles to its asymptotic limit $\left\langle h, f^{\text {in }}\right\rangle e$ (see the top-right diagram), while in complete agreement with Theorem 3 , the deviation $\left\|e^{-r t} G_{U_{m}}(t) f^{\text {in }}-\left\langle h, f^{\text {in }}\right\rangle e\right\|_{[m]}$ decreases exponentially as $t$ increases (see the bottom-left diagram).

A crucial role in the analysis is played by (33). It ensures that most of the mass of the daughter particles is concentrated in smaller particles, [6]. A large class of fragmentation kernels, which can be considered to be a discrete equivalent of the homogeneous kernels in continuous fragmentation, satisfying (33) is presented in the next example.

EXAMPLE 3. Assume that $b_{k, n}$ can be written as

$$
b_{k, n}=\zeta(n) h\left(\frac{k}{n}\right), \quad 1 \leq k \leq n-1, \quad n \in \mathbb{N},
$$

where $h$ is a Riemann integrable function on $[0,1]$ and $\zeta(n)$ is an appropriate sequence that ensures that (3) is satisfied. By (3), we have

$$
1=\zeta(n)(n-1) \sum_{k=1}^{n-1} \frac{k}{n} h\left(\frac{k}{n}\right) \frac{1}{n-1} .
$$



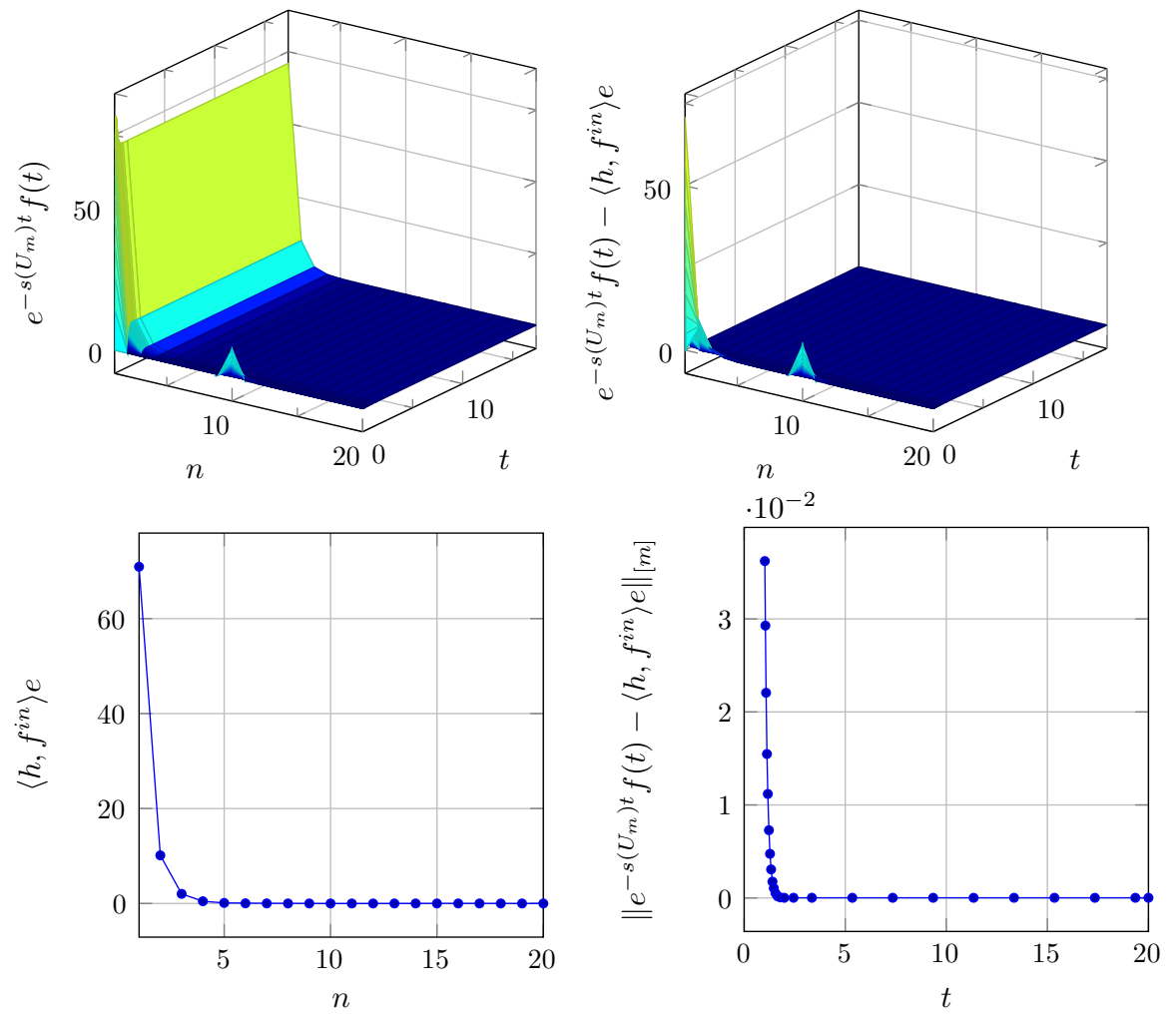

Figure 1. Long-time behaviour of (40). The semigroup solution $f(t)=G_{U_{m}}(t) f^{\text {in }}$ of (40) (top-left); the asymptotic error $e^{-s\left(U_{m}\right) t} f(t)-\left\langle h, f^{\text {in }}\right\rangle e$ (top-right); the asymptotic mass distribution $\left\langle h, f^{\text {in }}\right\rangle e$ (bottom-left) and the evolution of the asymptotic error $\left\|e^{-s\left(U_{m}\right) t} f(t)-\left\langle h, f^{\text {in }}\right\rangle e\right\|_{[m]}$, for $t \geq 1$ (bottom-right)

Since

$$
\frac{k-1}{n-1} \leq \frac{k}{n} \leq \frac{k}{n-1}
$$

for $1 \leq k \leq n$, we have

$$
\lim _{n \rightarrow \infty} \sum_{k=1}^{n} \frac{k}{n} h\left(\frac{k}{n}\right) \frac{1}{n-1}=\int_{0}^{1} z h(z) \mathrm{d} z
$$

and thus

$$
\lim _{n \rightarrow \infty}(n-1) \zeta(n)=\frac{1}{\int_{0}^{1} z h(z) \mathrm{d} z} .
$$


Therefore,

$$
\begin{aligned}
\lim _{n \rightarrow \infty} \sum_{k=1}^{n-1}\left(\frac{k}{n}\right)^{p} b_{k, n} & =\lim _{n \rightarrow \infty} \zeta(n)(n-1) \sum_{k=1}^{n-1}\left(\frac{k}{n}\right)^{p} h\left(\frac{k}{n}\right) \frac{1}{n-1} \\
& =\frac{\int_{0}^{1} z^{p} h(z) \mathrm{d} z}{\int_{0}^{1} z h(z) \mathrm{d} z}<1 .
\end{aligned}
$$

Thus,

$$
\liminf _{n \rightarrow \infty} \frac{\Delta_{n}^{(p)}}{n^{p}}=\lim _{n \rightarrow \infty} \frac{n^{p}-\sum_{k=1}^{n-1} k^{p} b_{k, n}}{n^{p}}>0
$$

and hence (33) is satisfied.

We note that (46) is obviously satisfied by the binary uniform fragmentation

$$
b_{n, i}=\frac{2}{i-1}, \quad n=1, \ldots, i-1 .
$$

Another example is offered by the binary fragmentation written in terms of a symmetric infinite matrix $\left(\psi_{i, j}\right)_{i, j \geq 1}$ as

$$
\frac{\mathrm{d} f_{n}}{\mathrm{~d} t}=-\frac{1}{2} f_{n} \sum_{i=1}^{n-1} \psi_{i, n-i}+\sum_{i=n+1}^{\infty} \psi_{n, i-n} f_{i}, \quad n \geq 1,
$$

see $[4,16,42]$. Translating into our notation, we get

$$
b_{n, i}=\frac{\psi_{n, i-n}}{a_{i}}, \quad a_{n}=\frac{1}{2} \sum_{i=1}^{n-1} \psi_{i, n-i}, \quad i \geq 2, \quad 1 \leq n \leq i-1 .
$$

Typical cases in the polymer degradation are $\psi_{i, j}=(i+j)^{\beta}$ and $\psi_{i, j}=(i j)^{\beta}$. The first case gives $a_{n}=\frac{1}{2} n^{\beta}(n-1)$ and $b_{n, i}=\frac{2}{i-1}$, and hence, it is a uniform binary fragmentation (see the long-time behaviour of $G_{U_{m}}(t) f^{\text {in }}$, with $m=2, \beta=\frac{1}{10}$, $g_{n}=d_{n}=n^{1+\beta}$ and $f_{n}^{\text {in }}=\delta_{10, n} 10$, in Fig. 2). In the second case, we have

$$
b_{n, i}=\frac{n^{\beta}(i-n)^{\beta}}{a_{i}}=\frac{i^{2 \beta}}{a_{i}}\left(\frac{n}{i}\right)^{\beta}\left(1-\frac{n}{i}\right)^{\beta}
$$

and (46) is satisfied with $\zeta(n)=\frac{n^{2 \beta}}{a_{n}}$ and $h(z)=z^{\beta}(1-z)^{\beta}$.

A typical qualitative behaviour of $G_{U_{m}}(t) f^{\text {in }}$, with $m=2, \beta=\frac{1}{10}, d_{n}=g_{n}=$ $n^{1+\beta}$ and $f_{n}^{\text {in }}=\delta_{10, n} 10$, is shown in Fig. 3 .

EXAMPLE 4. On the other hand, the fragmentation process given by

$$
\begin{aligned}
& b_{1,2}=2, \quad \text { and } \quad b_{1, i}=b_{i-1, i}=1, \\
& b_{n, i}=0, \quad i \geq 2, \quad 2 \leq n \leq i-2,
\end{aligned}
$$

obviously does not satisfy (33) and, in fact, the corresponding semigroup is neither analytic, nor compact, see [6]. 


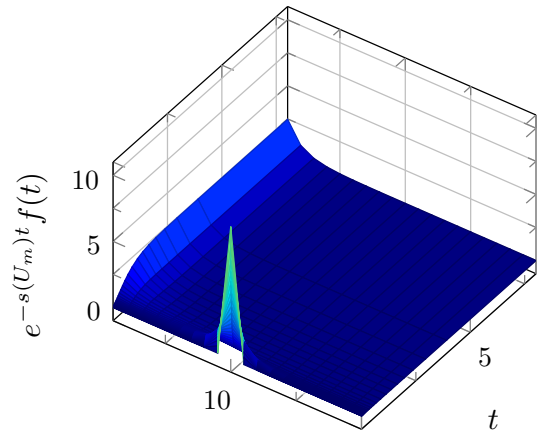

n

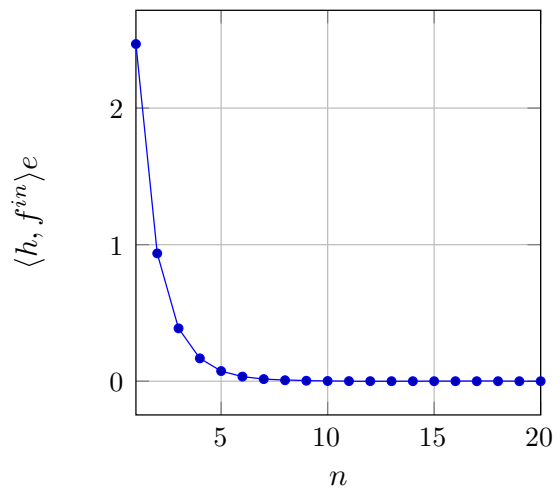

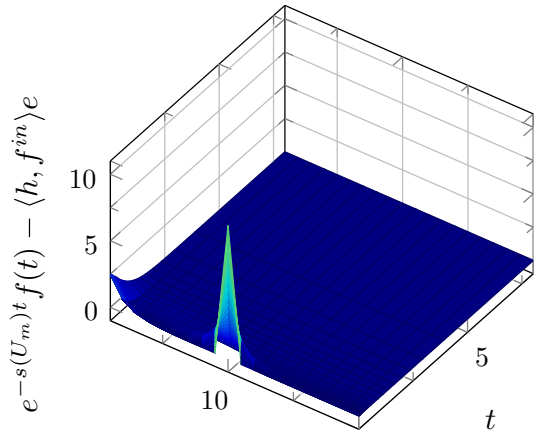

200

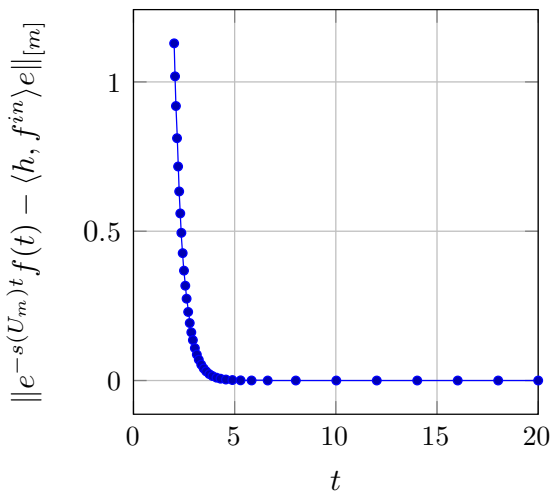

Figure 2. Long-time behaviour of (1), $\psi_{i, j}=(i+j)^{\beta}$. The semigroup solution $f(t)=G_{U_{m}}(t) f^{\text {in }}$ of (1) (top-left); the asymptotic error $e^{-s\left(U_{m}\right) t} f(t)-\left\langle h, f^{\text {in }}\right\rangle e$ (top-right); the asymptotic mass distribution $\left\langle h, f^{\text {in }}\right\rangle e$ (bottom-left) and the evolution of the asymptotic error $\left\|e^{-s\left(U_{m}\right) t} f(t)-\left\langle h, f^{\text {in }}\right\rangle e\right\|_{[m]}$, for $t \geq 1$ (bottom-right)

\section{Appendix: an alternative view at the model}

In Theorem 1, we have seen a regularizing role played by the diagonal operator induced by $\mathcal{A}$ even in the case not involving the full fragmentation operator. In many applications, however, (1) models a combination of two independent processes - the birth-and-death process and the fragmentation process and it is important to investigate when they exist irrespective of each other. In other words, we consider (1) as

$$
\frac{\mathrm{d}}{\mathrm{d} t} f=\mathcal{G} f+\mathcal{D} f+\mathcal{F} f, \quad f(0)=f^{\text {in }} .
$$

The pure birth-and-death problem

$$
\frac{\mathrm{d}}{\mathrm{d} t} f=\mathcal{V} f=\mathcal{G} f+\mathcal{D} f, \quad f(0)=f^{\text {in }}
$$

has been extensively analysed in the space $X_{0}$, see, e.g., [8, Chapter 7]. Its behaviour in $X_{m}$ creates, however, unexpected challenges. 

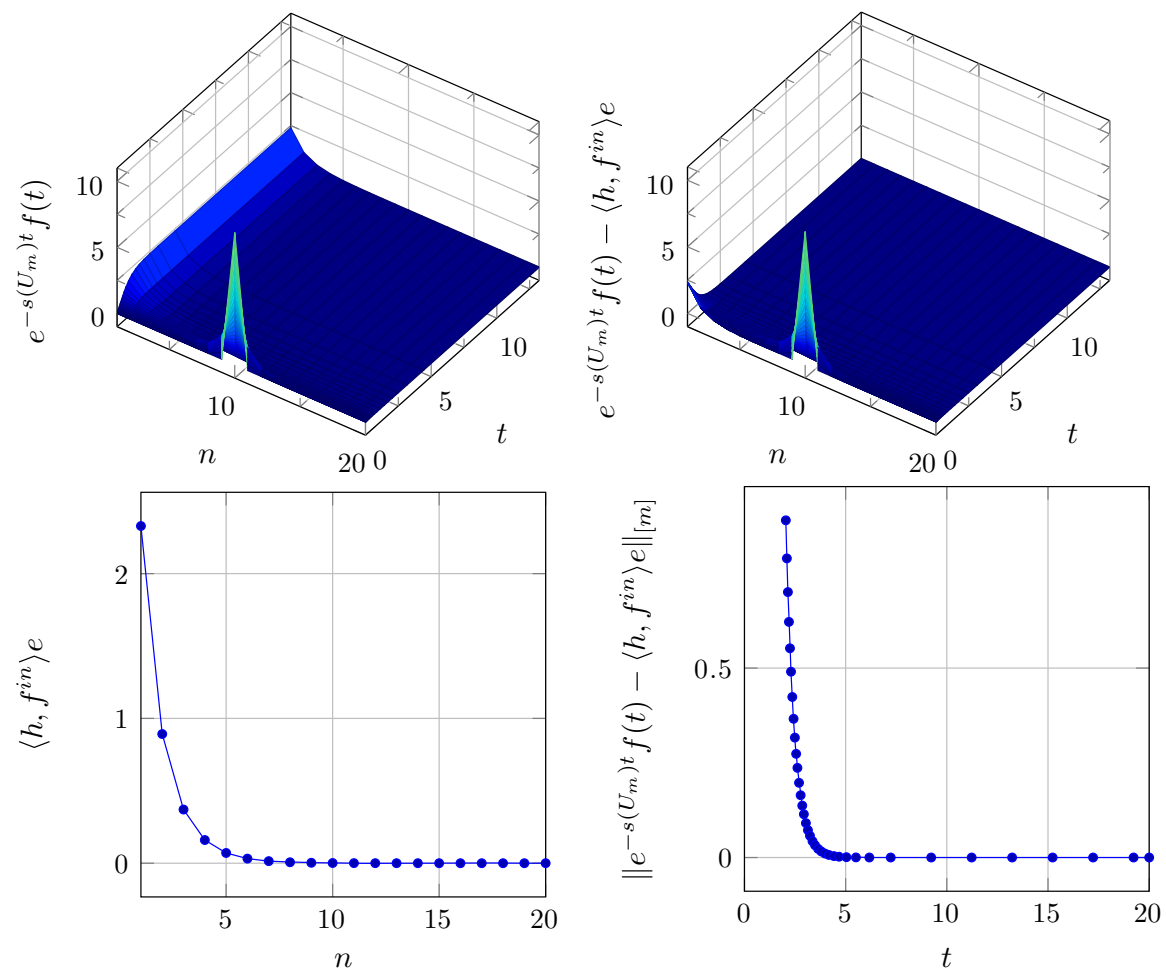

Figure 3. Long-time behaviour of (1), $\psi_{i, j}=(i j)^{\beta}$. The semigroup solution $f(t)=G_{U_{m}}(t) f^{\text {in }}$ of (1) (top-left); the asymptotic error $e^{-s\left(U_{m}\right) t} f(t)-\left\langle h, f^{\text {in }}\right\rangle e$ (top-right); the asymptotic mass distribution $\left\langle h, f^{\text {in }}\right\rangle e$ (bottom-left) and the evolution of the asymptotic error $\left\|e^{-s\left(U_{m}\right) t} f(t)-\left\langle h, f^{\text {in }}\right\rangle e\right\|_{[m]}$, for $t \geq 1$ (bottom-right)

EXAMPLE 5. If there is $C$ such that

$$
g_{n} \leq C n, \quad n \geq 1
$$

then there is a realization of the growth expression $\mathcal{G}$ that generates a $C_{0}$-semigroup in $X_{m}$. Indeed, this again follows from the Kato-Voigt theorem. We consider $\mathcal{G}$ as the perturbation of $\mathcal{G}^{0}$ by $\mathcal{G}^{-}$; that is, we introduce $G_{m}^{0}=\left.\mathcal{G}^{0}\right|_{D\left(G_{m}^{0}\right)}$, with

$$
D\left(G_{m}^{0}\right)=\left\{f \in X_{m} ; \mathcal{G}^{0} f \in X_{m}\right\}
$$

Then, as in (17), for $f \in D\left(G_{m}^{0}\right)$,

$$
\sum_{n=1}^{\infty} n^{m}\left[\left(G_{m}^{0}+G_{m}^{-}\right) f\right]_{n}=\sum_{n=0}^{\infty} n^{m} f_{n}\left(g_{n} \frac{(n+1)^{m}-n^{m}}{n^{m}}\right) \leq C^{\prime}\|f\|_{[m]},
$$


for some constant $C^{\prime}$. Hence, there is an extension of $G_{m}^{0}+G_{m}^{-}$generating a $C_{0^{-}}$ semigroup in $X_{m}$. On the other hand, if for some $c, C>0$

$$
c n^{q} \leq g_{n} \leq C n^{q}, \quad n \geq 1, q>1,
$$

then there is no realization of $\mathcal{G}$ with resolvent bounded in $X_{m}$ with $q \leq m+1$. Indeed, the resolvent of the generator, if it exists, must be given by (11),

$$
\left[R_{\lambda} f\right]_{n}=\sum_{i=1}^{n} \frac{f_{i}}{\lambda+g_{n}} \prod_{j=i}^{n-1} \frac{g_{j}}{\lambda+g_{j}}, \quad n \geq 1 .
$$

Let us fix $\lambda$. Then,

$$
\prod_{j=i}^{n-1} \frac{g_{j}}{\lambda+g_{j}} \geq g_{\lambda}:=\prod_{j=1}^{\infty} \frac{g_{j}}{\lambda+g_{j}}
$$

where $g_{\lambda} \neq 0$, and, for $f \in X_{m,+}$,

$$
\begin{aligned}
\left\|R_{\lambda} f\right\|_{[m]} & =\sum_{n=1}^{\infty} n^{m} \sum_{i=1}^{n} \frac{f_{i}}{\lambda+g_{n}} \prod_{j=i}^{n-1} \frac{g_{j}}{\lambda+g_{j}}=\sum_{i=1}^{\infty} f_{i} \sum_{n=i}^{\infty} \frac{n^{m}}{\lambda+g_{n}} \prod_{j=i}^{n-1} \frac{g_{j}}{\lambda+g_{j}} \\
& \geq g_{\lambda} \sum_{i=1}^{\infty} f_{i} \sum_{n=i}^{\infty} \frac{n^{m}}{\left(\lambda+g_{n}\right)} \geq g_{\lambda} C^{-1} \sum_{i=1}^{\infty} f_{i} \sum_{n=i}^{\infty} \frac{1}{n^{q-m}} .
\end{aligned}
$$

Hence, $R_{\lambda}$ is not bounded if (52) is satisfied and hence, in particular, there is no realization of $\mathcal{G}$ generating a $C_{0}$-semigroup in $X_{m}$. We note that for $q=2$ and $m=1$ we have a discrete version of the nonexistence result obtained in [11, Remark 2].

Let us return to the full birth-and-death model (48). As before, we introduce $V_{m}^{0}+$ $V_{m}^{1}:=G_{m}^{0}+D_{m}^{0}+G_{m}^{-}+D_{m}^{+}$on

$$
D\left(V_{m}^{0}\right)=\left\{f \in X_{m} ;\left(\mathcal{G}^{0}+\mathcal{D}^{0}\right) f \in X_{m}\right\} .
$$

We have

THEOREM 4. 1. If

$$
\limsup _{n \rightarrow \infty} \Gamma_{n} \leq C
$$

for some constant $C \in \mathbb{R}$, where

$$
\Gamma_{n}=g_{n}\left(\left(1+\frac{1}{n}\right)^{m}-1\right)-d_{n}\left(1-\left(1-\frac{1}{n}\right)^{m}\right),
$$

then there is an extension $V_{m}$ of $V_{m}^{0}+V_{m}^{1}$ that generates a quasicontractive semigroup $\left(G_{V_{m}}(t)\right)_{t \geq 0}$ on $X_{m}$. 
2. Condition (54) is satisfied if either

(a) (50) holds, or

(b) $\limsup _{n \rightarrow \infty} \frac{d_{n}}{g_{n}}>1$ and $d_{n}=O\left(n^{2}\right)$, or

(c) $\frac{d_{n}}{g_{n}} \geq 1+\frac{m^{\prime}-1}{n}$ for sufficiently large $n$ and $m^{\prime}>m$.

3. If any of the conditions of point 2 is satisfied, then $V_{m}=\overline{V_{m}^{0}+V_{m}^{1}}$.

Proof. Statement 1 of the theorem follows in a standard way as an application of the Kato-Voigt theorem as for $f \in D\left(V_{m}^{0}\right)_{+}$we have

$$
\sum_{n=1}^{\infty} n^{m}\left[\left(G_{m}^{0}+D_{m}^{0}+G_{m}^{-}+D_{m}^{+}\right) f\right]_{n}=\sum_{n=1}^{\infty} n^{m} f_{n} \Gamma_{n} .
$$

For statement 2, we observe that

$$
\begin{aligned}
& \left(1+\frac{1}{n}\right)^{m}-1=\frac{m}{n}+\frac{m(m-1)}{2 n^{2}}+O\left(\frac{1}{n^{3}}\right), \\
& 1-\left(1-\frac{1}{n}\right)^{m}=\frac{m}{n}-\frac{m(m-1)}{2 n^{2}}+O\left(\frac{1}{n^{3}}\right) .
\end{aligned}
$$

Thus, if 2(a) is satisfied, then the positive part of $\Gamma_{n}$ is bounded. If 2(b) is satisfied, then

$$
\Gamma_{n} \leq d_{n}\left(\frac{m(m-1)}{n^{2}}+O\left(\frac{1}{n^{3}}\right)\right)
$$

for sufficiently large $n$ and hence again $\Gamma_{n}$ is bounded from above. Finally, if 2(c) is satisfied, then

$$
\begin{aligned}
\Gamma_{n} & \leq g_{n}\left(\left(\frac{m(m-1)}{n^{2}}+O\left(\frac{1}{n^{3}}\right)\right)-\frac{m^{\prime}-1}{n}\left(\frac{m}{n}-\frac{m(m-1)}{2 n^{2}}+O\left(\frac{1}{n^{3}}\right)\right)\right) \\
& =\frac{g_{n}}{n^{2}}\left(m\left(m-m^{\prime}\right)+O\left(\frac{1}{n}\right)\right)
\end{aligned}
$$

and hence $\Gamma_{n}$ is negative for large $n$ and thus also bounded from above.

To prove the last statement, we use the approach of [8, Theorem 7.11], based on the extension technique, see [8, Theorem 6.22]. Let $f \in D\left(V_{m}\right)_{+}$. Then,

$$
\begin{aligned}
& \sum_{n=1}^{\infty} n^{m}\left(-\left(g_{n}+d_{n}\right) f_{n}+g_{n-1} f_{n-1}+d_{n+1} f_{n+1}\right) \\
& =\sum_{k=1}^{\infty} k^{m} f_{k} \Gamma_{k}+\lim _{n \rightarrow \infty}\left(-g_{n} f_{n}+d_{n+1} f_{n+1}\right) n^{m},
\end{aligned}
$$

where the limit exists. For honesty, it suffices to prove that for any $f \in D\left(V_{m}\right)_{+}$

$$
\lim _{n \rightarrow \infty}\left(-g_{n} f_{n}+d_{n+1} f_{n+1}\right) n^{m} \geq 0 .
$$


Assume, to the contrary, that for some $0 \leq f \in D\left(V_{m}\right)_{+}$, the limit is negative so that there exists $b>0$ such that

$$
\left(-g_{n} f_{n}+d_{n+1} f_{n+1}\right) n^{m} \leq-b,
$$

for all $n \geq n_{0}$ with large enough $n_{0}$. Thus, for $n \geq n_{0}$ we obtain by induction that for arbitrary $k$

$$
f_{n} \geq \frac{b}{g_{n}}\left(\sum_{i=0}^{k} \frac{1}{(n+i)^{m}} \prod_{j=1}^{i} \frac{d_{n+j}}{g_{n+j}}\right) \text {. }
$$

Because $k$ is arbitrary, we obtain

$$
f_{n} \geq \frac{b}{g_{n}}\left(\sum_{i=0}^{\infty} \frac{1}{(n+i)^{m}} \prod_{j=1}^{i} \frac{d_{n+j}}{g_{n+j}}\right), \quad n \geq n_{0} .
$$

Thus, if

$$
\sum_{n=1}^{\infty} \frac{n^{m}}{g_{n}}\left(\sum_{i=0}^{\infty} \frac{1}{(n+i)^{m}} \prod_{j=1}^{i} \frac{d_{n+j}}{g_{n+j}}\right)=+\infty
$$

(where we put $\prod_{j=1}^{0} \cdot=1$ ) is satisfied, then $\sum_{n=0}^{\infty} n^{m} f_{n}=+\infty$ which contradicts $f \in D\left(V_{m}\right)_{+}$.

Now, if (50) is satisfied, we have

$$
\sum_{n=1}^{\infty} \frac{n^{m}}{g_{n}}\left(\sum_{i=0}^{\infty} \frac{1}{(n+i)^{m}} \prod_{j=1}^{i} \frac{d_{n+j}}{g_{n+j}}\right) \geq \sum_{n=1}^{\infty} \frac{1}{g_{n}}=+\infty
$$

Similarly, if assumption 2(b) is satisfied, we have

$$
\sum_{n=1}^{\infty} \frac{n^{m}}{g_{n}}\left(\sum_{i=0}^{\infty} \frac{1}{(n+i)^{m}} \prod_{j=1}^{i} \frac{d_{n+j}}{g_{n+j}}\right) \geq \sum_{n=1}^{\infty} \frac{n^{m}}{g_{n}}\left(\sum_{i=n}^{\infty} \frac{1}{i^{m}}\right) \geq C \sum_{n=1}^{\infty} \frac{1}{n}=+\infty,
$$

where we used the integral estimate for the inner sum. Finally, if 2(c) is satisfied, we can write

$$
\sum_{n=1}^{\infty} \frac{n^{m}}{g_{n}}\left(\sum_{i=0}^{\infty} \frac{1}{(n+i)^{m}} \prod_{j=1}^{i} \frac{d_{n+j}}{g_{n+j}}\right) \geq \sum_{n=1}^{\infty} \frac{n^{m}}{g_{n}}\left(\sum_{i=0}^{\infty} \frac{1}{(n+i)^{m}} \prod_{j=1}^{i}\left(1+\frac{m^{\prime}-1}{n+j}\right)\right) .
$$

Now, as in the proof of Theorem 1, by the Stirling formula,

$$
\prod_{j=1}^{i}\left(1+\frac{m^{\prime}-1}{n+j}\right)=\frac{\Gamma\left(n+i+m^{\prime}\right) \Gamma(n+1)}{\Gamma\left(n+m^{\prime}\right) \Gamma(n+i+1)}=O\left(\left(\frac{n+i+m^{\prime}}{n+m^{\prime}}\right)^{m^{\prime}-1}\right)
$$

and the inner series diverges if the second condition of 2(c) is satisfied. 
By [6, Theorem 2.1], under standard assumptions on the fragmentation coefficients, $F_{m}=\overline{A_{m}+B_{m}}$ generates a quasicontractive $\left(G_{F_{m}}(t)\right)_{t \geq 0}$.

THEOREM 5. Assume the conditions of Theorem 2, item 1, and of Theorem 4, item 3, are satisfied. Then, $Y_{m}=\overline{V_{m}+F_{m}}$ and

$$
G_{Y_{m}}(t) f=\lim _{n \rightarrow \infty}\left(G_{V_{m}}\left(\frac{t}{n}\right) G_{F_{m}}\left(\frac{t}{n}\right)\right)^{n} f, \quad f \in X_{m},
$$

uniformly on bounded time intervals.

Proof. First, we observe that $D\left(V_{m}\right) \cap D\left(F_{m}\right) \supset D\left(G_{m}^{0}\right) \cap D\left(D_{m}^{0}\right) \cap D\left(A_{m}^{0}\right)$ and the latter is dense in $X_{m}$. Next, we see that

$$
\begin{aligned}
& {\left[\lambda I-\left(V_{m}+F_{m}\right)\right] D\left(V_{m}\right) \cap D\left(A_{m}\right)} \\
& \quad \supset\left[\lambda I-\left(V_{m}+F_{m}\right)\right] D\left(G_{m}^{0}\right) \cap D\left(D_{m}^{0}\right) \cap D\left(A_{m}\right) \\
& \quad=\left[\lambda I-\left(T_{m}+G_{m}^{-}+D_{m}^{+}+B_{m}\right)\right] D\left(T_{m}\right) .
\end{aligned}
$$

Since $\overline{\left(T_{m}+G_{m}^{-}+D_{m}^{+}+B_{m}, D\left(T_{m}\right)\right)}$ is the generator a semigroup, $\left[\lambda I-\left(T_{m}+\right.\right.$ $\left.\left.G_{m}^{-}+D_{m}^{+}+B_{m}\right)\right] D\left(T_{m}\right)$ is dense in $X_{m}$ for sufficiently large $\lambda$. Indeed, if $f \in X_{m}$, then $f=\left(\lambda I-\overline{T_{m}+G_{m}^{-}+D_{m}^{+}+B_{m}}\right) u$ for some $u \in D\left(\overline{T_{m}+G_{m}^{-}+D_{m}^{+}+B_{m}}\right)$ and $u=\lim _{n \rightarrow \infty} u_{n}$ with $u_{n} \in D\left(T_{m}\right)$ and

$$
\lim _{n \rightarrow \infty}\left(T_{m}+G_{m}^{-}+D_{m}^{+}+B_{m}\right) u_{n}=\overline{T_{m}+G_{m}^{-}+D_{m}^{+}+B_{m}} u .
$$

But then $f=\lim _{n \rightarrow \infty}\left(\lambda u_{n}-\left(T_{m}+G_{m}^{-}+D_{m}^{+}+B_{m}\right) u_{n}\right)$; that is, $f \in$ $\overline{\left[\lambda I-\left(T_{m}+G_{m}^{-}+D_{m}^{+}+B_{m}\right)\right] D\left(T_{m}\right)}$.

Since both $\left(G_{V_{m}}(t)\right)_{t \geq 0}$ and $\left(G_{F_{m}}(t)\right)_{t \geq 0}$ are quasicontractive, [35, Corollary 3.5.5] implies that $\overline{V_{m}+F_{m}}$ is the generator of a quasicontractive semigroup. Now

$$
\begin{aligned}
\lambda I & -Y_{m}=\lambda I-\overline{T_{m}+G_{m}^{-}+D_{m}^{+}+B_{m}} \\
& =\lambda I-\overline{\left(D_{m}^{0}+D_{m}^{+}+G_{m}^{-}+G_{m}^{0}\right)+\left(A_{m}+B_{m}\right)} \subset \lambda I-\overline{V_{m}+F_{m}}
\end{aligned}
$$

and, since both $Y_{m}$ and $\overline{V_{m}+F_{m}}$ are generators, we must have $Y_{m}=\overline{V_{m}+F_{m}}$. Then, (58) follows from [35, Corollary 3.5.5].

Open Access. This article is distributed under the terms of the Creative Commons Attribution 4.0 International License (http://creativecommons.org/licenses/by/4.0/), which permits unrestricted use, distribution, and reproduction in any medium, provided you give appropriate credit to the original author(s) and the source, provide a link to the Creative Commons license, and indicate if changes were made.

Publisher's Note Springer Nature remains neutral with regard to jurisdictional claims in published maps and institutional affiliations. 


\section{REFERENCES}

[1] Azmy S. Ackleh and Ben G. Fitzpatrick. Modeling aggregation and growth processes in an algal population model: analysis and computations. Journal of Mathematical Biology, 35(4):480-502, 1997.

[2] Wolfgang Arendt and Abdelaziz Rhandi. Perturbation of positive semigroups. Arch. Math. (Basel), 56(2):107-119, 1991.

[3] Ovide Arino. Some spectral properties for the asymptotic behaviour of semigroups connected to population dynamics. SIAM Review, 34(3):445-476, 1992.

[4] John M. Ball and Jack Carr. The discrete coagulation-fragmentation equations: existence, uniqueness, and density conservation. J. Statist. Phys., 61(1-2):203-234, 1990.

[5] Jacek Banasiak, Luke O. Joel, and S. Shindin. Analysis and simulations of the discrete fragmentation equation with decay. Mathematical Methods in the Applied Sciences, 41(16):6530-6545, 2018.

[6] Jacek Banasiak. Global classical solutions of coagulation-fragmentation equations with unbounded coagulation rates. Nonlinear Anal. Real World Appl., 13(1):91-105, 2012.

[7] Jacek Banasiak. Transport processes with coagulation and strong fragmentation. Discrete Contin. Dyn. Syst. Ser. B, 17(2):445-472, 2012.

[8] Jacek Banasiak and Luisa Arlotti. Perturbations of positive semigroups with applications. Springer Monographs in Mathematics. Springer-Verlag London, Ltd., London, 2006.

[9] Jacek Banasiak and Wilson Lamb. Coagulation, fragmentation and growth processes in a size structured population. Discrete Contin. Dyn. Syst. Ser. B, 11(3):563-585, 2009.

[10] Jacek Banasiak and Wilson Lamb. The discrete fragmentation equation: semigroups, compactness and asynchronous exponential growth. Kinet. Relat. Models, 5(2):223-236, 2012.

[11] Jacek Banasiak, Suares C. Oukouomi Noutchie, and Ryszard Rudnicki. Global solvability of a fragmentation-coagulation equation with growth and restricted coagulation. J. Nonlinear Math. Phys., 16(suppl. 1):13-26, 2009.

[12] Jacek Banasiak, Katarzyna Pichór, and Ryszard Rudnicki. Asynchronous exponential growth of a general structured population model. Acta Appl. Math., 119:149-166, 2012.

[13] George I Bell and Ernest C Anderson. Cell growth and division: I. a mathematical model with applications to cell volume distributions in mammalian suspension cultures. Biophysical Journal, 7(4):329-351, 1967.

[14] Mao Cai, Boyd F Edwards, and Hongtao Han. Exact and asymptotic scaling solutions for fragmentation with mass loss. Physical Review A, 43(2):656, 1991.

[15] Philippe Clément, Henk J. A. M. Heijmans, Sigurd B. Angenent, Cornelis J. van Duijn, and Ben de Pagter. One-parameter semigroups, volume 5 of CWI Monographs. North-Holland Publishing Co., Amsterdam, 1987.

[16] Fernando P. da Costa. Existence and uniqueness of density conserving solutions to the coagulationfragmentation equations with strong fragmentation. J. Math. Anal. Appl., 192(3):892-914, 1995.

[17] Pierre Degond, Jian-Guo Liu, and Robert L. Pego. Coagulation-Fragmentation Model for Animal Group-Size Statistics. J. Nonlinear Sci., 27(2):379-424, 2017.

[18] Odo Diekmann, Henk JAM Heijmans, and Horst R Thieme. On the stability of the cell size distribution. Journal of Mathematical Biology, 19(2):227-248, 1984.

[19] Marie Doumic and Miguel Escobedo. Time asymptotics for a critical case in fragmentation and growth-fragmentation equations. Kinet. Relat. Models, 9(2):251-297, 2016.

[20] Marie Doumic Jauffret and Pierre Gabriel. Eigenelements of a general aggregation-fragmentation model. Math. Models Methods Appl. Sci., 20(5):757-783, 2010.

[21] Ronald L. Drake. A general mathematical survey of the coagulation equation. In G.M. Hidy and J.R. Brock, editors, Topics in Current Aerosol Research, International Reviews in Aerosol Physics and Chemistry, pages $201-376$. Pergamon, 1972.

[22] Boyd F Edwards, Mao Cai, and Hongtao Han. Rate equation and scaling for fragmentation with mass loss. Physical Review A, 41(10):5755-5757, 1990.

[23] Klaus-Jochen Engel and Rainer Nagel. One-parameter semigroups for linear evolution equations, volume 194 of Graduate Texts in Mathematics. Springer-Verlag, New York, 2000. 
[24] Klaus-Jochen Engel and Rainer Nagel. A short course on operator semigroups. Universitext. Springer, New York, 2006.

[25] W. Gautschi. Some elementary inequalities relating to the gamma and incomplete gamma function. J.Math. and Phys., 38:77-81, 1959.

[26] Shay Gueron and Simon A Levin. The dynamics of group formation. Mathematical biosciences, 128(1):243-264, 1995.

[27] George A Jackson. A model of the formation of marine algal flocs by physical coagulation processes. Deep Sea Research Part A. Oceanographic Research Papers, 37(8):1197-1211, 1990.

[28] Philippe Laurençot and Benoît Perthame. Exponential decay for the growth-fragmentation/celldivision equation. Commun. Math. Sci., 7(2):503-510, 2009.

[29] Z. Aleksander Melzak. The effect of coalescence in certain collision processes. Quart. Appl. Math., $\mathrm{XI}(2): 231-234,1953$.

[30] Philippe Michel, Stéphane Mischler, and Benoît Perthame. General relative entropy inequality: an illustration on growth models. J. Math. Pures Appl. (9), 84(9):1235-1260, 2005.

[31] Stéphane Mischler and J. Scher. Spectral analysis of semigroups and growth-fragmentation equations. Ann. Inst. H. Poincaré Anal. Non Linéaire, 33(3):849-898, 2016.

[32] Hans Müller. Zur allgemeinen theorie ser raschen koagulation. Fortschrittsberichte über Kolloide und Polymere, 27(6):223-250, 1928.

[33] Akira Okubo. Dynamical aspects of animal grouping: swarms, schools, flocks, and herds. Advances in biophysics, 22:1-94, 1986.

[34] Akira Okubo and Simon A. Levin. Diffusion and ecological problems: modern perspectives, volume 14 of Interdisciplinary Applied Mathematics. Springer-Verlag, New York, second edition, 2001.

[35] Amnon Pazy. Semigroups of linear operators and applications to partial differential equations, volume 44 of Applied Mathematical Sciences. Springer-Verlag, New York, 1983.

[36] Benoît Perthame. Transport equations in biology. Frontiers in Mathematics. Birkhäuser Verlag, Basel, 2007.

[37] Benoît Perthame and Lenya Ryzhik. Exponential decay for the fragmentation or cell-division equation. J. Differential Equations, 210(1):155-177, 2005.

[38] James W Sinko and William Streifer. A model for population reproducing by fission. Ecology, 52(2):330-335, 1971.

[39] Louise Smith, Wilson Lamb, Matthias Langer and Adam McBride. Discrete fragmentation with mass loss. J. Evol. Equ., 12(1):181-201, 2012.

[40] Marian Smoluchowski. Drei Vortrage über Diffusion, Brownsche Bewegung und Koagulation von Kolloidteilchen. Zeitschrift fur Physik, 17:557-585, 1916.

[41] Marian Smoluchowski. Versuch einer mathematischen theorie der koagulationskinetik kolloider lösungen. Zeitschrift fuer physikalische Chemie, 92:129 - 168, 2010.

[42] Robert M. Ziff and E.D. McGrady. Kinetics of polymer degradation. Macromolecules, 19(10):2513$2519,1986$. 


\section{J. Banasiak}

Department of Mathematics and Applied Mathematics University of Pretoria

\section{Pretoria}

South Africa

and

Institute of Mathematics

Technical University of Łódź

Łódź

Poland

E-mail: jacek.banasiak@up.ac.za

L.O. Joel and S. Shindin

School of Mathematics, Statistics and Computer Science

University of Kwazulu-Natal

Durban

South Africa

E-mail: oluwaseyejoel@gmail.com

S. Shindin

E-mail: shindins@ukzn.ac.za 